\title{
DIFFERENTIABLE REPRESENTATIONS. I: INDUCED REPRESENTATIONS AND FROBENIUS RECIPROCITY
}

\author{
BY \\ JOHAN F. AARNES
}

\begin{abstract}
In this paper we give the construction of the adjoint and the co-adjoint of the restriction functor in the category of differentiable $G$-modules, where $G$ is a Lie group. Stated in terms of representation theory this means that two types of induced representations are introduced, both differing from the classical definition of differentiably induced representation given by Bruhat. The Frobenius reciprocity theorem is shown to hold.

The main part of the paper is devoted to obtaining suitable realizations of the spaces of the induced representations. It turns out that they may be given as $E_{K}(G, E)$ and $E_{K}^{\prime}(G, E)$ respectively, i.e. as certain spaces of $K$-invariant differentiable functions or distributions on $G$. This makes it possible to establish a rather complete duality theory. In the last part we consider the relationship to some of Bruhat's work. In particular his Frobenius theorem is shown to be a direct consequence of the tensor-product machinery we employ. We also offer a result on inducing in stages.
\end{abstract}

1. Introduction. Differentiable representations of Lie groups were introduced by F. Bruhat in his fundamental and by now classical work [2]. Exploiting the interplay between unitary representations and their associated differentiable representations he obtained deep and useful criteria for the irreducibility of induced unitary representations. Central in his discussions is the space of intertwining forms between two representations. He proves a version of the Frobenius reciprocity theorem for his concept of differentiably induced representations in terms of intertwining forms. This result may be regarded as the solution (in a particular case) of a universal problem. In our terminology it is equivalent to the construction of a "quasi-adjoint" for the restriction functor $F \rightarrow F_{K}$, where $F$ is a differentiable $G$-module, $G$ a Lie group, and $K$ a closed subgroup of $G$.

Starting with Frobenius [4] the history of the Frobenius reciprocity theorem is long and interesting. An important factor in this development were the works of Mackey [14], [15] and [16] where he gives general and profound versions for unitary representations. More recently Moore [20], Rieffel [22] and

Received by the editors May 29, 1974.

AMS (MOS) subject classifications (1970). Primary 22E50, 22D30, 43A65; Secondary 43A95, 46F05, 46M05.

Key words and phrases. Lie group, differentiable representation, induced representation, Frobenius reciprocity, adjoint functor, co-adjoint functor, tensor product. 
Rigelhof [23] have all given versions of the theorem from a more general point of view. Specifically, if $E$ and $F$ are $K$ - and $G$-modules respectively, then the restriction functor $F \rightarrow F_{K}$ will (under various conditions on $E$ and $F$ ) have an adjoint $E \rightarrow{ }^{G} E$ and a co-adjoint $E \rightarrow E^{G}$. I.e.

$$
L_{G}\left({ }^{G} E, F\right) \cong L_{K}\left(E, F_{K}\right) \quad \text { and } \quad L_{G}\left(F, E^{G}\right) \cong L_{K}\left(F_{K}, E\right) .
$$

In particular Rigelhof succeeded in constructing an adjoint and a co-adjoint in the category of continuous locally convex $G$-modules. It is however, well known that in the form stated here, the Frobenius theorem is false for unitary representations. Therefore, in the spirit of Bruhat, we find it natural to seek a solution to the problem for differentiable representations. Using the algebraic machinery developed by Rieffel and Rigelhof we define differentiable induced and co-induced representations, in a natural way and show that the corresponding functors $E \rightarrow$ ${ }^{G} E$ and $E \rightarrow E^{G}$ are adjoint and co-adjoint for the restriction functor, respectively.

The spaces ${ }^{G} E$ and $E^{G}$ are, as defined, somewhat unwieldy. However, due to the many nice properties of spaces of differentiable functions and distributions we are able to give rather satisfactory realizations of ${ }^{G} E$ and $E^{G}$. Specifically, we show that $E^{G}$ may be realized as the space $E_{K}(G, E)$ of $E$-valued $K$-invariant differentiable functions on $G$, whereas ${ }^{G} E$ (in most important cases) may be realized as $E_{K}^{\prime}(G, E)=$ the space of $E$-valued $K$-linear distributions with compact support modulo $K$. On the basis of these realizations we obtain a fairly complete duality for the induced and the co-induced representations. If $\pi$ is a differentiable representation of $K$ on $E$, $\check{\pi}$ the contragredient representation on $\check{E}$, then (under suitable conditions on $E$ ) we have $\left({ }^{G} E\right)^{-} \cong(\check{E})^{G}$ and ${ }^{G}(\check{E})=\left(E^{G}\right)^{\complement}$. These results, and the realization ${ }^{G} E=E_{K}^{\prime}(G, E)$ mentioned above are definitive improvements of the corresponding results of Rigelhof for continuous representations. The reason being of course the strong effect of differentiability assumptions.

Our concept of induced (and co-induced) differentiable representation differs from that of Bruhat. There is however, a rather interesting connection. In particular, if $E$ is the dual of a reflexive Fréchet space then the adjoint ${ }^{G} E$ is in fact a quasi-adjoint for the restriction functor. This complements Bruhat's own result, where he restricts both $E$ and $F$ to be Fréchet spaces. We are also able to show that Bruhat's Frobenius theorem is an almost direct consequence of the tensor product machinery we employ.

This paper is the first of a sequence. The organization of the material is as follows. In $\S 2$ we give the basic facts concerning the specific types of tensor products which we are going to deal with. In $\S 3$ we present the main facts concerning differentiable representations and the distribution form of such represen- 
tations. The theory of vector-valued distributions on Lie groups, their convolutions etc. is by now well established. For general background we refer to [29] (Appendix 2), the introductory chapters of [2], or [26] and [27]. In $\$ 4$ we define our versions of the induced and the co-induced differentiable representations, and in the following section we prove the Frobenius reciprocity theorem for them. In $\S 6$ we give the realizations of the spaces ${ }^{G} E$ and $E^{G}$. $\S 7$ is devoted to the proof of the realization of ${ }^{G} E$. Here we also compute the dual of $G_{E}$, which is the basis for the duality mentioned above, and which is presented in $\S 8$. In $\S 9$ we take up the discussion of the relationship to Bruhat's work, and prove and extend his Frobenius result. Finally, in $\S 10$ we offer a result on inducing in stages and some concluding observations.

In a forthcoming paper we will take up the question of irreducibility of unitary and differentiable representations. If $U$ is a unitary representation of $K$ on a Hilbert space $X$, and $\pi$ the associated differentiable representation on $E=$ $X_{\infty}$, we find the triplet ${ }^{G} \breve{E}, X^{G}$ and $E^{G}$ (where $X^{G}$ is the Hilbert space of the unitary induced representation $U^{G}$ ) a very useful tool.

In another paper we will show that the so-called duality theorem for automorphic functions [5] is a fairly direct consequence of our Frobenius theorem.

Although the present work is in the spirit of representation theory, we find the language of modules and category theory convenient; and shall use it freely. Specifically, and to establish the rules of the game; if $\rho$ is a representation of a group (or an algebra with convolution $x \rightarrow \tilde{x}$ ) $X$ on a space $E$, we realize $E$ as a left (right) $X$-module under the action $x a=\rho(x) a\left(a x=\rho\left(x^{-1}\right) a\right)$ (or $\rho(\widetilde{x}) a$ if $X$ is an algebra) ( $x \in X, a \in E$ ). Conversely, to any left or right $X$-module we obtain a representation of $X$ on $E$ in the obvious way. If $E$ is a locally convex space and also a separately continuous left (right) $X$-module, then we can make $E^{\prime}$ (= the dual of $E$ ), into a right (left) module by $\left\langle a, a^{\prime} x\right\rangle=\left\langle x a, a^{\prime}\right\rangle$ $\left(\left\langle a, x a^{\prime}\right\rangle=\left\langle a x, a^{\prime}\right\rangle\right)$. The dual module of $E$ is the submodule $\check{E}$ of $E^{\prime}$ consisting of those elements $a^{\prime} \in E^{\prime}$ such that $x \rightarrow a^{\prime} x \quad\left(x \rightarrow x a^{\prime}\right)$ is continuous of $X$ into $E_{b}^{\prime}$. If $\rho$ is a representation of $X$ on $E$, then the contragredient representation $\check{\rho}$ of $X$ is the representation associated with the dual module, viz. $\check{\rho}(x)={ }^{t} \rho\left(x^{-1}\right)$ (or ${ }^{t} \rho(\widetilde{x})$ if $X$ is an algebra) where ${ }^{t} A$ is the transpose of the continuous linear operator $A$ on $E$, restricted to $\breve{E}$.

With respect to notation we shall with few exceptions follow Warner [29]. We will however, write $D(G), E(G), D^{\prime}(G)$ and $E^{\prime}(G)$ for the differentiable functions on $G$ with compact support; the differentiable functions, the distributions and the distributions with compact support, respectively.

If $G$ is a Lie group we denote by $\&$ its Lie algebra, and by $Ð(ङ)$ the universal enveloping algebra of the complexification of $\&$. We identify $\mathcal{S}(\&)$ with the right invariant differential operators on $G$. 
All locally convex spaces are assumed to be Hausdorff. Our results are stated for Lie groups. Using the device of Bruhat in [3] , that any connected locally compact group is a projective limit of Lie groups (Yamabe's theorem), they may be extended to connected locally compact groups satisfying the second axiom of countability.

2. Generalities on tensor products. In this section we collect and present, mostly without proofs, the results concerning tensor products which will be needed in this work. For proofs and a more complete discussion the reader is refered to [7], [23] and [1].

Let $X$ be a group or an algebra over $\mathrm{C}$, and let $E$ and $F$ be vector spaces over C. Suppose that $X$ acts to the right on $E$ and to the left on $F$, i.e. that $E(F)$ is a right (left) $X$-module. A bilinear map $\omega: E \times F \rightarrow H$ where $H$ is a vector space over $\mathbf{C}$ is $X$-balanced if

$$
\omega(a x, b)=\omega(a, x b)
$$

for all $x \in X, a \in E$ and $b \in F$. The linear space of all $X$-balanced bilinear maps $\omega: E \times F \rightarrow H$ is denoted by $B_{X}(E, F ; H)$. If $H=\mathrm{C}$ we write $B_{X}(E, F)$.

Let $\chi$ denote the canonical bilinear map of $E \times F$ into $B_{X}(E, F)^{*}$. I.e. $\chi(a, b) \omega=\omega(a, b) \quad\left(\omega \in B_{X}(E, F)\right)$, and $\chi$ is $X$-balanced. Let $E \otimes_{X} F$ denote the linear span of the range of $\chi$. We write $a \otimes_{X} b$ (or just $a \otimes b$ if no confusion is possible) for the element $\chi(a, b)$. Hence $E \otimes_{X} F$ consists of all finite sums $\Sigma a_{i} \otimes_{X} b_{i}$ with $a_{i} \in E, b_{i} \in F$. We refer to $E \otimes_{X} F$ as the $X$-tensor product of $E$ and $F$.

We have the following basic algebraic fact.

PPRoposition 2.1. Each $X$-balanced bilinear map $\omega$ on $E \times F$ into $H$ determines a unique linear operator $T_{\omega}$ of $E \otimes_{X} F \rightarrow H$ such that $\omega=T_{\omega} \circ \chi$. The correspondence $\omega \rightarrow T$ is an isomorphism of $B_{X}(E, F)$ onto $L\left(E \otimes_{X} F, H\right)$.

The property of Proposition 2.1 characterizes the $X$-tensor product of $E$ and $F$ up to isomorphism. Here is another approach to the construction. Let $E \otimes F=$ $E \otimes_{\mathrm{C}} F$ be the usual tensor product of $E$ and $F$ over the complex numbers. Let $N$ be the subspace of $E \otimes F$ generated by elements of the form $a x \otimes b-a \otimes x b$ $(x \in X, a \in E, b \in F)$.

Proposition 2.2 The spaces $E \otimes F / N$ and $E \otimes_{X} F$ are naturally isomorphic.

Now suppose that $E$ and $F$ are locally convex spaces. As for ordinary tensor products it is possible to introduce several locally convex topologies on $E \otimes_{X} F$, each making continuous linear maps on $E \otimes_{X} F$ correspond to bilinear $X$-balanced maps on $E \times F$ satisfying specific continuity requirements. The problem 
here, however, is that these topologies on $E \otimes_{X} F$ need not be Hausdorff, reflecting the fact that $N$ need not be closed in the corresponding topology on $E \otimes F$. We shall therefore pass to the "Hausdorffication" of $E \otimes F$, i.e. to the quotient space $\left.E \otimes_{X} F /\{0\}\right\}^{-}$, where $\{0\}^{-}$denotes the closure of $\{0\}$ in the topology in question.

As for ordinary tensor products there are unique topologies $\pi$ and $\beta$ on $E \otimes_{X} F$ such that continuous linear maps $T: E \otimes_{X} F \rightarrow H$ define continuous and hypocontinuous bilinear maps on $E \times F \rightarrow H$. $\pi$ and $\beta$ will be called the projective and the inductive tensor product topology. Let $E \otimes_{X}^{\pi} F$ (resp. $E \otimes_{X}^{\beta}$ $F)$ denote the quotient of $E \otimes_{X} F$ modulo the closure of $\{0\}$ with respect to $\pi$ (resp. $\beta)$. Let $B_{X}^{c}(E, F ; H)$ (resp. $B_{X}(E, F ; H)$ ) denote the space of continuous (resp. hypocontinuous) $X$-balanced bilinear maps of $E \times F$ into $H$. We have

Proposition 2.3. Let $H$ be a locally convex Hausdorff space. The correspondence $\omega=T \circ \chi$ defines a linear isomorphism between $L\left(E \otimes_{X}^{\pi} E, H\right)$ and $B_{X}^{c}(E, F ; H)\left(L\left(E \otimes_{X}^{\beta} F, H\right)\right.$ and $\left.B_{X}(E, F ; H)\right)$. Moreover, equicontinuous subsets of $L\left(E \otimes_{X}^{\pi} F, H\right)\left(\right.$ resp. $\left.L\left(E \otimes_{X}^{\beta} F, H\right)\right)$ correspond to equicontinuous (resp. equihypocontinuous) subsets of $B_{X}^{c}(E, F ; H)\left(\right.$ resp. $\left.B_{X}(E, F ; H)\right)$. The topology $\pi(\beta)$ is the topology of uniform convergence on equicontinuous (equihypocontinuous) subsets of $B_{X}(E, F ; H)$. If $\bar{N}$ is the closure of $N$ in $E \otimes F$ with respect to $\pi(\beta)$ then we have a topological isomorphism between $E \otimes_{\pi} F / \bar{N}\left(E \otimes_{\beta} F / \bar{N}\right)$ and $E \otimes_{X}^{\pi} F\left(E \otimes_{X}^{\beta} F\right)$.

Remark 2.4. The Hausdorff condition on $H$ is crucial here, if $T$ is a continuous linear map of $M$ into $H, T^{-1}\{0\}$ is closed so $T$ defines a continuous linear map on the quotient $M /\{0\}^{-}$. For some further discussion of the Hausdorff property on $X$-tensor products, see [1] where examples and counterexamples may be found. Now let $X=A$ be a topological algebra, and suppose that the action of $A$ on $E$ and $F$ is at least separately continuous. Assume that $A$ contains a group $G$ such that $[G]$ is dense in $A$. (This is, for instance, the case if $G$ is a Lie group and $A=E^{\prime}(G)$ via the natural imbedding $x \rightarrow \epsilon_{x}$ of $G$ into $E^{\prime}(G)$.)

Proposition 2.5. Under the assumptions above we have, for $\tau=\pi$ or $\beta$ :

$$
E \otimes_{G}^{\tau} F \cong E \otimes_{A}^{\tau} F .
$$

Proof. Let $\chi: E \times F \rightarrow E \otimes_{G}^{\tau} F$ be the canonical bilinear map. $\chi$ is $G$-balanced and separately continuous for both topologies. Hence, under the given assumptions $\chi$ is $A$-balanced and the result follows easily.

We shall from now on assume that $X=G$ is a topological group, and we shall also assume that the action of $G$ on $E$ and $F$ is at least separately continuous. $G$ acts naturally on $F^{\prime}$ to the right, viz: $\left\langle b, b^{\prime} x\right\rangle=\left\langle x b, b^{\prime}\right\rangle(x \in G, b \in F$, 
$\left.b^{\prime} \in F^{\prime}\right)$. Let $L_{G}\left(E, F_{b}^{\prime}\right)$ be the space of continuous $G$-linear maps $m$ of $E$ into $F_{b}^{\prime}$, i.e. $m(a x)=(m a) x(a \in E)$. Let $L_{G}^{\epsilon}\left(E, F_{b}^{\prime}\right)$ denote the subspace of those $m$ 's that map bounded subsets of $E$ into equicontinuous subsets of $F_{b}^{\prime}$. If $\varphi \in$ $\left(E \otimes_{G}^{\beta} E\right)^{\prime}$ define $m_{\varphi}: E \rightarrow F_{b}^{\prime}$ by $\left\langle b, m_{\varphi}(a)\right\rangle=\varphi(a \otimes b)$.

PROPOSITION 2.6. The map $\Phi: \varphi \rightarrow m_{\varphi}$ is a linear isomorphism of $\left(E \otimes_{G}^{\beta} F\right)^{\prime}$ onto $L_{G}^{\epsilon}\left(E, F_{b}^{\prime}\right)$. $\Phi$ is a topological isomorphism with respect to the topology of bi-bounded convergence on $\left(E \otimes_{G}^{\beta} F\right)^{\prime}$ and the topology of bounded convergence on $L_{G}^{\epsilon}\left(E, F_{b}^{\prime}\right)$, respectively,

Proof. The proof is routine and is omitted.

Later on we shall need associativity of tensor products with inductive topologies, and we shall also need associativity of completed tensor products. To make this go through we have to impose certain conditions on the tensor products. Let us say that $E \otimes_{G}^{\beta} F$ is saturated (with respect to bounded sets) if each bounded set $Q \subseteq E \otimes_{G}^{\beta} F$ is contained in the closure of the convex, balanced hull of a set $A \otimes B$, where $A$ and $B$ are bounded in $E$ and $F$ respectively.

Let $D, E$ and $F$ be locally convex spaces, and let $K$ and $H$ be topological groups, such that $K$ acts to the right on $D$ and to the left on $E$ and $H$ acts to the right on $E$ and to the left on $F$. We assume that the actions of $K$ and $H$ on $E$ commute, viz $(k a) h=k(a h)(k \in K, h \in H, a \in E)$. Observe that $K$ acts naturally on $E \otimes_{H} F$ to the left, and $H$ acts to the right on $D \otimes_{K} E$; viz: $k(a \otimes b)=$ $k a \otimes b$ and $(d \otimes a) h=d \otimes a h$, the definitions being made through the natural bilinear maps. These actions are separately continuous on $F \otimes_{H}^{\beta} F$ and $D \otimes_{K}^{\beta} E$.

Proposition 2.7. Let $D, E, F ; K$ and $H$ be as above and suppose that $E \otimes_{H} F$ and $D \otimes_{K} E$ are saturated. Then there is a natural topological isomorphism

$$
D \otimes_{K}^{\beta}\left(E \otimes_{H}^{\beta} F\right) \cong\left(D \otimes_{K}^{\beta} E\right) \otimes_{H}^{\beta} F .
$$

Proof. Rigelhof's Proposition 7 [23] does not cover this case, since he considers a different topology on the tensor products. His proof may be modified however, and can be used as a basis for a proof. The complete argument is somewhat tedious, and may be found in [1].

To obtain associativity for the completed tensor products we need the following.

Lemma 2.8. Suppose $G$ acts to the right on $E$ and to the left on $F$. Suppose moreover that $E_{0}$ is a dense $G$-invariant subspace of $E$ such that any bounded subset of $E$ is contained in the closure of a bounded subset of $E_{0}$. Then any hypocontinuous $G$-balanced bilinear form $\omega$ on $E_{0} \times F$ has a unique hypocontinuous G-balanced bilinear extension $\tilde{\omega}$ to $E \times F$. 
Corollary 2.9. Let, $E, E_{0}, F$ and $G$ be as above. Then the natural map of $E_{0} \otimes_{G}^{\beta} F$ into $E \otimes_{G}^{\beta} F$ is injective and a topological isomorphism onto a dense subspace.

Proofs. The proof of the lemma proceeds along traditional lines and is omitted. Let $\chi$ be the canonical bilinear map of $E \times F$ into $E \otimes_{G} F$. The restriction of $\chi$ to $E_{0} \times F$ is hypocontinuous and determines a continuous linear map $i$ of $E_{0} \otimes_{G}^{\beta} F$ into $E \otimes_{G}^{\beta} F$. Since the spaces are Hausdorff it follows by the Hahn-Banach theorem and the lemma that $i$ is injective. The range of $i$ is clearly dense in $E \otimes_{G}^{\beta} F$ since $E_{0}$ is dense in $E$ and $\chi$ is separately continuous. Finally, if $H$ is an equihypocontinuous subset of $B_{G}\left(E_{0}, F\right)$ then the set $\widetilde{H}=$ $\{\tilde{\omega}: \omega \in H\}$ is equihypocontinuous in $B_{G}(E, F)$. The result follows.

Let $E \bar{\otimes}_{G} F$ denote the completion of $E \otimes_{G}^{\beta} F$. Now let $D, E, F ; K$ and $H$ be as in Proposition 2.7 and assume in addition that $D, E$ and $F$ are complete. We may extend the action of $K$ on $E \otimes_{H}^{\beta} F$ to $E \bar{\otimes}_{H} F$ by continuity, each $k \in K$ defines a continuous automorphism $u \rightarrow k u\left(u \in E \bar{\otimes}_{H} F\right)$ of $E \bar{\otimes}_{H} F$. Note however that without extra assumptions the maps $k \rightarrow k u$ need not be continuous for all $u \in E \bar{\otimes}_{H} F$. However, this is not necessary for the next result to hold. Let us say that the completed product $E \bar{\otimes}_{H_{*}} F$ is saturated (with respect to bounded sets) if each bounded set $Q$ in $E \bar{\otimes}_{H}^{*} F$ is contained in the closure (in $E \bar{\otimes}_{H} F$ ) of the convex, balanced hull of a set $A \otimes B$, where $A$ and $B$ are bounded in $E$ and $F$ respectively. Clearly $E \bar{\otimes}_{H} F$ is saturated if and only if $E \otimes_{H}^{\beta} F$ is saturated and each bounded subset of $E \bar{\otimes}_{H} F$ is contained in the closure of a bounded subset of $E \otimes_{H}^{\beta} F$.

Proposition 2.10. Let $D, E, F ; K$ and $H$ be as above with $D, E$ and $F$ complete. Suppose that $D \bar{\otimes}_{K} E$ and $E \bar{\otimes}_{H} F$ are saturated. Then we have a natural topological isomorphism

$$
D \bar{\otimes}_{K}\left(E \bar{\otimes}_{H} F\right) \cong\left(D \bar{\otimes}_{K} E\right) \bar{\otimes}_{H} F .
$$

Proof. By Corollary $2.9\left(D \otimes_{K}^{\beta} E\right) \otimes_{H}^{\beta} F$ carries the relative topology of $\left(D \bar{\otimes}_{K} E\right) \otimes_{H}^{\beta} F$ and is dense. On the other hand $D \otimes_{K}^{\beta}\left(E \otimes_{H}^{\beta} F\right)$ carries the relative topology of $D \otimes_{K}^{\beta}\left(E \bar{\otimes}_{H} F\right)$ and is dense. Now $D \otimes_{K}^{\beta}\left(E \otimes_{H}^{\beta} F\right) \cong$ $\left(D \otimes_{K}^{\beta} E\right) \otimes_{H}^{\beta} F$ by Proposition 2.7. The result follows.

REMARK 2.11. Proposition 2.10 is the basis for the result on "inducing in stages" to be given in $\S 10$ of this work, and our proof of Bruhat's Frobenius result, given in $\S 9$.

3. Differentiable representations. Distribution form of a representation. In this section we will recall the basic fact and properties concerning differentiable representations, and prove a few results for them. 
Let $G$ be a Lie group, countable at infinity and let $\rho$ be a continuous representation of $G$ on a complete locally convex space $E$. For $a \in E$ define the function $\hat{a}: G \rightarrow E$ by

$$
\hat{a}(x)=\rho(x) a \quad(x \in G) .
$$

The vector $a$ is differentiable if $\hat{a} \in E(G, E)$. The subspace of differentiable vectors in $E$ is denoted by $E_{\infty}$ and is dense in $E$. The linear map $A: a \rightarrow \hat{a}$ is a linear isomorphism of $E_{\infty}$ onto a closed subspace $E_{G}$ of $E(G, E)$. We place on $E_{\infty}$ the relative topology of $E_{G}$ as a subspace of $E(G, E)$. Clearly, if $E$ is a metric space, $E_{\infty}$ is a metric space.

The representation $\rho$ is differentiable if $E=E_{\infty}$ with coincidence of topologies, equivalently $A: E \rightarrow E(G, E)$ is a topological isomorphism onto $E_{G}$. Another formulation is that the operators $\rho(D)(D \in \mathcal{J}(\&))$ are continuous on $E_{\infty}$ when $E_{\infty}$ is given the relative topology from $E$ [2], [29] .

In general $E_{\infty}$ is a $\rho$-invariant subspace of $E$. The corresponding representation $\rho_{\infty}$ of $G$ on $E_{\infty}$ is continuous and differentiable, and $\left(E_{\infty}\right)_{\infty}=E_{\infty}$ with coincidence of topologies. The representation $\rho_{\infty}$ is topologically irreducible if and only if $\rho$ is topologically irreducible [2].

Proposition 3.1. If $E$ is a reflexive Fréchet space, then $E_{\infty}$ is a reflexive Fréchet space.

Proof. If $E$ is a Fréchet space, so is $E(G, E)$ and therefore also the closed subspace $E_{G}$. Now $E(G, E) \cong E(G) \hat{\otimes} E$ so it follows from [7, §3, Proposition 13] that $E(G, E)$ is semireflexive if $E$ is. A closed subspace of a semireflexive space is semireflexive [12, 20.2(ii)] and a Fréchet space is reflexive if it is semireflexive. The result follows.

Let $\rho$ be a differentiable representation of $G$ on $E$, and let $\check{\rho}$ be the contragredient representation on $\check{E}$. Then $\check{E}=E^{\prime}$ and $\check{\rho}(D)$ is continuous on $E_{b}^{\prime}$ for all $D \in \cong(\&)$ [2]. Hence, if $E_{b}^{\prime}$ is complete $\check{\rho}$ is a differentiable representation. In any case $\check{\rho}$ extends to a continuous and differentiable representation on the completion of $\check{E}$.

Since $E$ is complete we have $E(G, E) \cong E(G) \hat{\otimes} E$. Any $S \in E^{\prime}(G)$ gives rise to a continuous linear operator $S \otimes 1$ on $E(G) \otimes E$ into $E$. We have $(S \otimes 1)$ - $(h \otimes a)=S(h) a(h \in E, a \in E)$ since the map $(h, a) \rightarrow S(h) a$ obviously is continuous and bilinear. The corresponding continuous linear map of $E(G, E)$ into $E$ will be denoted by $S^{\prime}$. We use the notation

$$
S^{\prime} f=\int_{G} f(x) d S(x) \quad(f \in E(G, E)) .
$$

This is appropriate, for if $S=\mu$, where $\mu$ is a measure of compact support then $\mu^{\prime} f=\int_{G} f(x) d \mu(x)$. Here the right-hand side is defined as a vector-valued integral in the usual sense. 
Let $S$ and $f$ be as above. We may then define their convolutions $S * f$ and $f * S$ in a natural way [29].

Proposition 3.2. $S * f$ and $f * S$ are $C^{\infty}$-functions and $G$ with values in $E$ given by

$$
\begin{aligned}
& (S * f)(x)=\int_{G} f\left(y^{-1} x\right) d S(y), \\
& (f * S)(x)=\int_{G} f\left(x y^{-1}\right) \delta_{G}\left(y^{-1}\right) d S(y) .
\end{aligned}
$$

The bilinear maps $(f, S) \rightarrow f * S$ and $(f, S) \rightarrow S * f$ of $E(G, E) \times E^{\prime}(G)$ into $E(G, E)$ are hypocontinuous.

We assume all the usual properties of convolutions to be known, at least their verification is standard procedure and is left to the reader. The hypocontinuity property does not seem to be stated in the literature in this generality however, and will be proved here. We do this by exploiting the natural isomorphism $\Gamma$ between $E(G, E)$ and $L\left(E^{\prime}, E\right)$, where the latter is given the topology of uniform convergence on bounded (i.e. compact) subsets of $E^{\prime}$.

$$
\Gamma(f)(S)=S^{\prime}(f) \quad\left(f \in E(G, E), S \in E^{\prime}\right) .
$$

If $h \in E$ we put $\widetilde{h}(x)=h\left(x^{-1}\right) \delta_{G}\left(x^{-1}\right)$, and define $\check{S}(h)=S(\widetilde{h}), \tilde{S}(h)=S\left(\widetilde{h} \delta_{G}\right)$. (If $S=\mu$ this is consistent with the definition $\widetilde{\mu}(A)=\mu\left(A^{-1}\right)$, where $A$ is a Borel set.)

Lemma 3.3. For $S, T \in E^{\prime}, m \in L\left(E^{\prime}, E\right)$ we define

$$
\begin{aligned}
& (S \circ m)(T)=m(\tilde{S} * T), \\
& (m \circ S)(T)=m(T * \check{S}) .
\end{aligned}
$$

This makes $L\left(E^{\prime}, E\right)$ into an $E^{\prime}$-bimodule. The bilinear maps $(S, m) \rightarrow S \circ m$ and $(S, m) \rightarrow m \circ S$ are hypocontinuous.

Proof. The fact that (3.4) and (3.5) define left and right actions of $E$ on $L\left(E^{\prime}, E\right)$ follows directly from the relations

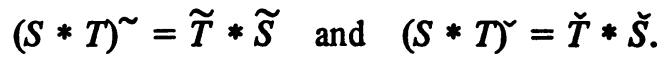

We prove that $(S, m) \rightarrow m \circ S$ is hypocontinuous. For the left action the proof is similar and is omitted. Observe first that the map $S \rightarrow \check{S}$ is an isomorphism of $E$ onto itself, so the bilinear map $(T, S) \rightarrow T * \check{S}$ is jointly continuous of $E^{\prime} \times E^{\prime}$ into $E^{\prime}[28]$.

Let $C$ be a compact subset of $E^{\prime}$ and let $V$ be a neighbourhood of 0 in $E$. Then

$$
W_{C, V}=\left\{m \in L\left(E^{\prime}, E\right): m(C) \subseteq V\right\}
$$


is a standard neighbourhood of 0 in $L\left(E^{\prime}, E\right)$. First, let $B$ be a bounded subset of $L\left(E^{\prime}, E\right)$. Since $E$ is barrelled $B$ is equicontinuous so there is a neighbourhood $U_{1}$ of 0 in $E^{\prime}$ such that $B\left(U_{1}\right) \subseteq V$.

Choose the neighbourhood $U$ of 0 in $E^{\prime}$ such that $T * \check{S}$ is in $U_{1}$ for all $T \in$ $C$ when $S$ is in $U$. Then $(m \circ S)(T)=m(T * \check{S})$ is in $V$ for all $m \in B, T \in C$ and $S \in U$. Hence $m \circ S \in W_{C, V}$ for all $m \in B, S \in U$. Next, let $W_{C, V}$ be as above and let $A$ be a closed bounded subset of $E$. Then $A$ is compact and $C_{1}=\{T * \check{S}$ : $T \in C, S \in A\}$ is compact by joint continuity. Hence, if $m \in W_{C_{1}, V}$ then $m(T * \check{S}) \in V$ for all $T \in C, S \in A$. Hence $m \circ S \in W_{C, V}$ for all $S \in A$ when $m \in W_{C_{1}, V}$. The proof is complete.

LEMma 3.4. $\Gamma: E(G, E) \rightarrow L\left(E^{\prime}, E\right)$ is an isomorphism between $E^{\prime}$-bimodules:

$$
\begin{aligned}
& \Gamma(S * f)=S \circ \Gamma(f) \\
& \Gamma(f * S)=\Gamma(f) \circ S
\end{aligned}
$$

Proof. $\Gamma(S * f)(T)=T^{\prime}(S * f)=(\tilde{S} * T)^{\prime}(f)=\Gamma(f)(\tilde{S} * T)=(S \circ \Gamma(f))(T)$ for all $T \in E^{\prime}$. This proves (3.6).

$$
\Gamma(f * S)(T)=T^{\prime}(f * S)=(T * \check{S})^{\prime}(f)=\Gamma(f)(T * \check{S})=(\Gamma(f) \circ S)(T)
$$

which proves (3.7).

Proposition 3.2 now follows immediately from Lemmata 3.3 and 3.4.

We now return to the differentiable representation $\rho$ of $G$ on $E$. Recall the isomorphism $a \rightarrow \hat{a}$ which identifies $E$ with the subspace $E_{G}$ of $E(G, E)$. We wish to realize $E$ as an $E^{\prime}$-module. The main point here is to observe that $E_{G}$ is invariant with respect to convolution with elements in $E^{\prime}$. Suppose first that $A: E \rightarrow F$ is a continuous linear map of $E$ into another complete locally convex space $F$. For any $f \in E(G, E)$ the composite map $A \circ f$ will belong to $E(G, F)$. We easily obtain that

$$
S^{\prime}(A \circ f)=A\left(S^{\prime} f\right) \quad\left(S \in E^{\prime}\right) .
$$

Indeed, it suffices to establish (3.8) for functions $f$ of the type $h \otimes a$ ( $h \in E$, $a \in E)$, and here it is immediate.

Now let $a \in E, S \in E$ :

$$
(\hat{a} * S)(x)=\int_{G} \hat{a}\left(x y^{-1}\right) \delta_{G}\left(y^{-1}\right) d S(y)=\int_{G} \rho(x) \hat{a}\left(y^{-1}\right) \delta_{G}\left(y^{-1}\right) d S(y)
$$

which equals $\rho(x) \breve{S}^{\prime}(\hat{a})$ by (3.8). So we get $\rho(x) \check{S}^{\prime}(\hat{a})=\left(\check{S}^{\prime}(\hat{a})\right)^{\hat{n}}(x)$. Hence

$$
\hat{a} * S=\left(\check{S}^{\prime}(\hat{a})\right)
$$

which proves the asserted invariance. We now define $\rho(S) a=S^{\prime}(\hat{a})$. 
Corollary 3.5. $S \rightarrow \rho(S)$ is a representation of $E^{\prime}$ on $E$, and the bilinear map $(S, a) \rightarrow \rho(S) a$ is hypocontinuous.

Proof. Follows immediately from (3.9) and Proposition 3.2.

The homomorphism $\rho: E^{\prime} \rightarrow L(E)$ is called the distribution form of the representation of $G$. Observe that $\rho\left(\epsilon_{x}\right)=\rho(x)$, where $\epsilon_{x}(\varphi)=\varphi(x)(\varphi \in E)$.

Remark 3.6. Suppose $E$ is a continuous left (right) $G$-module. We say that $E$ is a differentiable left (right) $G$-module if the associated representation is differentiable. Let $E$ (resp. $F$ ) be a differentiable left (right) $G$-module with associated representation $\rho(\lambda)$. I.e. $\rho(x) a=x a, \lambda(x) b=b x^{-1} \quad(x \in G, a \in E, b \in$ $F)$. We then define $S a=\rho(S) a, b S=\lambda(\widetilde{S}) b$ for $S \in E$. Clearly $E(F)$ becomes a left (right) $E^{\prime}$-module, and the bilinear maps $(S, a) \rightarrow S a,(b, S) \rightarrow b S$ are hypocontinuous. We also have $\epsilon_{x} a=x a$ and $b \epsilon_{x}=b x$.

For later reference we include the following facts.

Lemma 3.7. Let $E(F)$ be a left (right) differentiable G-module, and let $\omega: F \times E \rightarrow H$ be a separately continuous $G$-balanced bilinear map. Then $\omega$ is $E$-balanced when $E(F)$ is considered as a left (right) $E$-module.

Proof. The assertion follows immediately from the fact that $N=\left[\epsilon_{x}\right.$ : $x \in G]$ is a dense linear subspace of $E^{\prime}$. A more algebraic argument runs as follows: Define $F_{b}(a)=\omega(b, a)=G_{a}(b)$. Then $F_{b}\left(G_{a}\right)$ is continuous of $E(F)$ into $H$. By assumption on $\omega$ we have $\left(F_{b} \circ \hat{a}\right)(x)=\left(G_{a} \circ \hat{b}\right)\left(x^{-1}\right)$. Hence, if $S \in$ $E^{\prime}$ we get:

$$
\begin{aligned}
\omega(b, S a) & =F_{b}(S a)=F_{b}\left(S^{\prime} \hat{a}\right)=S^{\prime}\left(F_{b} \circ \hat{a}\right)=S^{\prime}\left(\left(G_{a} \circ \hat{b}\right) \sim \delta_{G}\right) \\
& =\widetilde{S}^{\prime}\left(G_{a} \circ \hat{b}\right)=G_{a}\left(\widetilde{S}^{\prime} \hat{b}\right)=G_{a}(b S)=\omega(b S, a) .
\end{aligned}
$$

There is an obvious analogue for intertwining operators between differentiable representations on modules. We omit the proof.

Lemma 3.8. Let $\rho, \lambda$ be differentiable representations of $G$ on $E, F$ respectively. Then an intertwining operator $A: E \rightarrow F$ also intertwines the distribution forms of $\rho$ and $\lambda$.

4. The induced and the co-induced representation. Let $G$ be a Lie group, countable at infinity and let $K$ be a closed subgroup. Once and for all we fix the following set of actions. $K$ acts to the left on functions on $G$ and to the right on distributions, viz: $(f \in E(G))$ (resp. $D(G)),\left(S \in E^{\prime}(G)\right)$ (resp. $D^{\prime}(G)$ )

$$
\begin{aligned}
& (k f)(x)=f(x k), \\
& (S k)(f)=S(k f) \quad(k \in K, x \in G) .
\end{aligned}
$$

$G$ acts to the right on functions and to the left on distributions: 


$$
\begin{aligned}
& (f z)(x)=f(z x), \\
& (z S)(f)=S(f z) .
\end{aligned}
$$

All these actions are jointly continuous and makes $E(G)$ (resp. $D(G)$ ) into a differentiable $(K, G)$-module, and $E^{\prime}(G)$ (resp. $D^{\prime}(G)$ ) into a differentiable $(G, K)$ module.

These actions satisfy the following relations, whose verification is immediate. Let $x \in G$ :

$$
\begin{aligned}
& x f=f * \check{\epsilon}_{x}, \quad f x=\widetilde{\epsilon}_{x} * f, \\
& x S=\epsilon_{x} * S, \quad S x=S * \epsilon_{x} .
\end{aligned}
$$

Moreover $\widetilde{\epsilon}_{x}=\epsilon_{x-1}$ and $\check{\epsilon}_{x}=\delta_{G}\left(x^{-1}\right) \epsilon_{x-1}$.

Throughout this section we assume that $\pi$ is a differentiable representation of $K$ on a complete locally convex space $E$.

(1) The induced representation. It will be convenient to regard $E$ as a left $K$-module, and we write $k a=\pi(k) a(k \in K, a \in E)$. We now employ the notation and results of $\S 2 . E^{\prime}$ is a right $K$-module so we can form the $K$-tensor product $E \otimes_{K} E$. We equip it with the inductive tensor product topology. Let ${ }^{G} E$ denote the (Hausdorff) completion of $E^{\prime} \otimes_{K} E$. Let $x \in G$; the bilinear form

$$
\omega_{x}(S, a)=x S \otimes a \quad\left(S \in E^{\prime}, a \in E\right)
$$

of $E^{\prime} \times E$ into $E^{\prime} \otimes_{K} E$ is evidently $K$-balanced and hypocontinuous. It therefore defines a continuous linear map ${ }^{G} \pi(x)$ of $E^{\prime} \otimes_{K} E$ into itself, viz:

$$
G_{\pi(x)} \sum S_{i} \otimes a_{i}=\sum x S_{i} \otimes a_{i}
$$

for all such finite sums. ${ }^{G} \pi(x)$ extends by continuity to all of ${ }^{G} E$. It is clearly an automorphism and ${ }^{G} \pi$ is a representation of $G$ on ${ }^{G} E$. We call ${ }^{G} \pi$ the induced representation of $G$ on ${ }^{G} E$.

Proposition 4.1. ${ }^{G} \pi$ is a differentiable representation.

Proof. We first verify that the action $(x, u) \rightarrow{ }^{G} \pi(x) u$ of $G$ on ${ }^{G} E$ is jointly continuous. Let $C$ be a compact subset of $G$. We will show that the family $\left\{{ }^{G} \pi(x): x \in C\right\}$ is equicontinuous. By Proposition 2.3 it suffices to show that $\left\{\omega_{x}: x \in C\right\}$ is equihypocontinuous of $E^{\prime} \times E$ into $E^{\prime} \otimes_{K} E$. So let $W=$ $H^{0}$ be a neighbourhood of 0 in $E^{\prime} \otimes_{K} E$, where $H$ is an equihypocontinuous subset of $B_{K}\left(E^{\prime}, E\right)$. Let $A$ be a bounded and closed subset of $E^{\prime}$. Then $A$ is compact, so by the joint continuity of the action $(x, S) \rightarrow x S$ it follows that $A_{1}=$ $\{x S: x \in C, S \in A\}$ is a compact subset of $E^{\prime}$. Hence there is a neighbourhood $V$ in $E$ such that $|b(T, a)| \leqslant 1$ for all $T \in A_{1}, a \in V$ and $b \in H$. Consequently, 
for all $x \in C, S \in A$ and $a \in V$ :

$$
\left|\omega_{x}(S, a) b\right|=|(x S \otimes a) b|=|b(x S, a)| \leqslant 1
$$

for all $b \in H$. So $\omega_{x}(A \times V) \subseteq W$ for all $x \in C$. Similarly, if $B$ is a bounded subset of $E$ we find a neighbourhood $U$ of 0 in $E$ such that $\omega_{x}(U \times B) \subseteq W$ for all $x \in C$. The crucial fact is again the joint continuity of the action $(x, S)$ $\rightarrow x S$. So $\left\{\omega_{x}: x \in C\right\}$ is equihypocontinuous and our first assertion follows.

The map $x \rightarrow{ }^{G} \pi(x) u$ of $G$ into ${ }^{G} E$ is clearly continuous for each $u \in{ }^{G} E$. Indeed, by the local equicontinuity of $G_{\pi}$ this follows from the fact that $x \rightarrow$ $x S \otimes a$ is continuous for each $S \in E$ and $a \in E$. Hence ${ }^{G} \pi$ is a jointly continuous representation.

The action $(x, S) \rightarrow x S$ of $G$ on $E^{\prime}$ is differentiable. We obtain

$$
\pi^{G}(D)(S \otimes a)=D S \otimes a \quad(D \in D(B))
$$

where $D S(\eta)=S\left(D^{\#} \eta\right)$ and $D^{\#}$ is the adjoint of $D$. It follows that each operator $G_{\pi(D)}$ is continuous on $E^{\prime} \otimes_{K} E$ and extends to a continuous operator on ${ }^{G} E$. This shows that ${ }^{G} \pi$ is a differentiable representation and completes the proof.

(2) The co-induced representation. For the construction it is convenient to regard $E$ as a right $K$-module. We then have $a k=\pi\left(k^{-1}\right) a(k \in K, a \in E)$. Let $E^{G}=L_{K}(E, E)$ denote the space of continuous $K$-linear maps $m: E \rightarrow E$, i.e. $m(S k)=m(S) k . E^{G}$ is evidently a closed subspace of $L_{b}(E, E)$ (which is complete) and is consequently a complete locally convex space in the relative topology.

We introduce a right action of $G$ on $L\left(E^{\prime}, E\right)$ by

$$
(m x)(S)=m(x S) \text {. }
$$

This action leaves $E^{G}$ invariant, indeed: $(m x)(S k)=m(x(S k))=m((x S) k)=$ $m(x S) k=(m x)(S) k$ which shows that $m \in E^{G}$ implies $m x \in E^{G}$. We therefore obtain a representation $\pi^{G}$ of $G$ on $E^{G}$ by restricting the action to $E^{G}$, viz:

$$
\left[\pi^{G}(x) m\right](S)=m\left(x^{-1} S\right) \text {. }
$$

We call $\pi^{G}$ the co-induced representation of $G$ on $E^{G}$.

Proposition 4.2. $\pi^{G}$ is a differentiable representation.

Proof. We sketch the argument. As in the proof of Proposition 4.1 one first shows that $\pi^{G}$ is jointly continuous. This is straightforward and the details are omitted. Let $D \in \Im(\&)$. One finds that $\left[\pi^{G}(D) m\right](S)=m\left(D^{\#} S\right)$ from which it easily follows that $\pi^{G}$ is differentiable. For later reference we include the following easy result, whose proof is omitted. 
Lemma 4.3. Let $S, T \in E^{\prime}, a \in E$ and $m \in E^{G}$ :

$$
\begin{gathered}
{ }_{\pi} \pi(S)(T \otimes a)=(S * T) \otimes a, \\
{\left[\pi^{G}(S) m\right](T)=m(\tilde{S} * T) .}
\end{gathered}
$$

REMARK 4.4. It should be observed that even without the assumption that $\pi$ is differentiable, the induced and co-induced representations are differentiable. We shall occasionally write ${ }_{K}^{G} E$ (resp. $E_{K}^{G}$ ) for ${ }^{G} E$ (resp. $E^{G}$ ) to indicate from which subgroup $K$ the inducing (resp. co-inducing) starts. One easily finds that $\underset{\{e\}}{G} \mathbf{C} \cong E^{\prime}$ and $\mathbf{C}_{\{e\}}^{G} \cong\left(E_{b}^{\prime}\right)^{\prime}=E$ with the regular actions.

LEMMA 4.5. Let $\pi$ be a differentiable representation of $G$ on $E$ (i.e. $K=G$ ). Then

$$
E^{\prime} \otimes_{G} E \cong E^{\prime} \bar{\otimes}_{G} E \cong E
$$

and

$$
L_{G}\left(E^{\prime}, E\right) \cong E \text {. }
$$

The proof is routine, and is omitted. It follows that ${ }_{G}^{G} E=E$ and $E_{G}^{G}=E$.

5. The Frobenius reciprocity theorem. Preserve the notation and assumptions from the preceding section. The theorem to be stated will be most precisely formulated in the language of category theory. If $H$ is a Lie group, let $\mathrm{C}_{l}^{\infty}(H)\left(\mathrm{C}_{r}^{\infty}(H)\right)$ denote the category of differentiable left (right) $H$-modules. There is a natural bijective functor inv: $C_{l}^{\infty}(H) \rightarrow C_{r}^{\infty}(H)$ which to each left $H$-module assigns the corresponding right $G$-module. Let $F$ be a left (right) differentiable $G$-module with associated representation $\lambda$. The restriction $F \rightarrow F_{K}$ is a functor

$$
\text { res: } \quad C_{l}^{\infty}(G) \rightarrow C_{l}^{\infty}(K) \quad\left(C_{r}^{\infty}(G) \rightarrow C_{r}^{\infty}(K)\right)
$$

which clearly commutes with inv. Similarly we see that ind: $E \rightarrow{ }^{G} E$ and co-ind: $E \rightarrow E^{G}$ define functors $C_{l}^{\infty}(K) \rightarrow C_{l}^{\infty}(G)\left(C_{r}^{\infty}(K) \rightarrow C_{r}^{\infty}(G)\right)$ that automatically commute with inv. Since ind and co-ind are functors we must have ${ }^{G} E \cong$ $G_{E_{1}}$ and $E^{G} \cong E_{1}^{G}$ if $E$ and $E_{1}$ are equivalent left (right) differentiable $K$-modules.

THEOREM 5.1. ind: $E \rightarrow{ }^{G} E$ is the adjoint and co-ind: $E \rightarrow E^{G}$ is the coadjoint functor of the restriction functor res: $F \rightarrow F_{K}$. I.e. there are natural isomorphisms (in the sense of category theory [18]):

$$
\begin{aligned}
& L_{G}\left({ }^{G} E, F\right) \cong L_{K}\left(E, F_{K}\right), \\
& L_{G}\left(F, E^{G}\right) \cong L_{K}\left(F_{K}, E\right) .
\end{aligned}
$$

Moreover, ind and co-ind are uniquely determined by (5.1) and (5.2) to within equivalence of differentiable G-modules. 
REMARK 5.2. It can also be shown that (5.1) and (5.2) are topological isomorphisms with respect to standard topologies.

Proof of Theorem. Suppose first that $E$ and $F$ are left $K$ - and $G$-modules respectively. To prove (5.1) let $A \in L_{K}\left(E, F_{K}\right)$ and consider the bilinear form $\omega_{A}(S, a)=\lambda(S) A a$ of $E^{\prime} \times E$ into $F$. Since $A$ is continuous it follows by Corollary 3.5 that $\omega_{A}$ is hypocontinuous. It is also $K$-balanced:

$$
\omega_{A}(S k, a)=\lambda(S k) A a=\lambda(S) \lambda(k) A a=\lambda(S) A \pi(k) a=\omega_{A}(S, k a)
$$

for $k \in K$ and where we have used (4.2). So $\omega_{A}$ determines a continuous linear map $A^{\prime}: E^{\prime} \otimes_{K} E \rightarrow F$ such that $A^{\prime}\left(\Sigma S_{i} \otimes a_{i}\right)=\Sigma \lambda\left(S_{i}\right) A a_{i}$ (finite sums); $A^{\prime}$ is $G$-linear, for if $x \in G$ then

$$
\begin{aligned}
A^{\prime}\left(G_{\left.\pi(x) \sum S_{i} \otimes a_{i}\right)}\right. & =A^{\prime} \sum x S_{i} \otimes a_{i}=\sum \lambda\left(x S_{i}\right) A a_{i}=\lambda(x) \sum \lambda\left(S_{i}\right) A a_{i} \\
& =\lambda(x) A^{\prime} \sum S_{i} \otimes a_{i}
\end{aligned}
$$

Hence $A^{\prime}$ extends to a continuous $G$-linear map of ${ }^{G} E$ into $F$. This map will also be denoted by $A^{\prime}$. The map $\varphi: A \rightarrow A^{\prime}$ of $L_{K}\left(E, F_{K}\right)$ into $L_{G}\left({ }^{G} E, F\right)$ obtained this way is linear. Now suppose $A^{\prime}=0$. Then $A a=\lambda\left(\epsilon_{e}\right) A a=$ $A^{\prime}\left(\epsilon_{e} \otimes a\right)=0$ for all $a \in E$ so $A=0$. Hence $\varphi$ is injective. Next, suppose $B \in$ $L_{G}\left({ }^{G} E, F\right)$ is arbitrary, and define $A a=B\left(\epsilon_{e} \otimes a\right)$ which certainly makes $A$ into a continuous $K$-linear map of $E$ into $F$. Hence $A \in L_{K}(E, F)$ and

$$
A^{\prime}(S \otimes a)=\lambda(S) A a=\lambda(S) B\left(\epsilon_{e} \otimes a\right)=B^{G} \pi(S)\left(\epsilon_{e} \otimes a\right)=B(S \otimes a)
$$

by Lemmata 3.8 and 4.3 . Consequently, by linearity and continuity $A^{\prime}=B$ so $\varphi$ is surjective and an isomorphism of $L_{K}\left(E, F_{K}\right)$ onto $L_{G}\left({ }^{G} E, F\right)$. This establishes (5.1) for left modules.

If $E$ and $F$ are right $K$ - and $G$-modules respectively we use the observation that the functor inv commutes with both res and ind. Property (5.1) for right modules then easily follows from the same result for left modules.

To prove (5.2) suppose first that $E$ and $F$ are right $K$ - and $G$-modules respectively. Let $A \in \operatorname{Hom}_{K}\left(F_{K}, E\right)$ and let $b \in F$. Define $\left(A^{\prime} b\right)(S)=A \lambda(\widetilde{S}) b$. Then $A^{\prime} b$ belongs to $L_{K}\left(E^{\prime}, E\right)=E^{G}$ since $S \rightarrow A \lambda(\widetilde{S}) b$ is continuous and

$$
\left(A^{\prime} b\right)(S k)=A \lambda\left(k^{-1}\right) \lambda(\widetilde{S}) b=\pi\left(k^{-1}\right) A \lambda(\widetilde{S}) b=\left(A^{\prime} b\right)(S) k .
$$

Hence $A^{\prime}$ maps $F$ into $E^{G}$ and is clearly linear. The continuity of $A^{\prime}$ follows almost immediately from Corollary 3.5 applied to $\lambda$. Finally, if $x \in G, b \in F$ then

$$
\begin{aligned}
A^{\prime}(b x)(S) & =\left(A^{\prime} \lambda\left(x^{-1}\right) b\right)(S)=\left(A \lambda(\tilde{S}) \lambda\left(x^{-1}\right)\right) b=A \lambda((x S) \sim b \\
& =\left(A^{\prime} b\right)(x S)=\left[\pi^{G}\left(x^{-1}\right) A^{\prime} b\right](S)
\end{aligned}
$$


for all $S \in E^{\prime}$. Hence $A^{\prime}(b x)=\left(A^{\prime} b\right) x$ which shows that $A^{\prime} \in L_{G}\left(F, E^{G}\right)$. The map $\psi: A \rightarrow A^{\prime}$ is linear, and if $A^{\prime}=0$ when $A b=A \lambda(e) b=\left(A^{\prime} b\right)\left(\epsilon_{e}\right)=0$ for all $b \in F$, so $A=0$. Hence $\psi$ is injective. To prove surjectivity let $B \in L_{G}\left(F, E^{G}\right)$ and define $A b=(B b)\left(\epsilon_{e}\right), b \in F$. It is easily seen that $A$ belongs to $L_{K}\left(F_{K}, E\right)$. Moreover $\left(A^{\prime} b\right)(S)=A \lambda(\widetilde{S}) b=(B \lambda(\widetilde{S}) b)\left(\epsilon_{e}\right)=\left(\pi^{G}(\tilde{S}) B b\right)\left(\epsilon_{e}\right)=(B b)(S)$ by Lemmata 3.8 and 4.3. Hence $A^{\prime}=B$ which shows that $\psi$ is an isomorphism and establishes (5.2) for right modules. The conversion to left modules is done as above.

The fact that $\varphi$ and $\psi$ are natural in the sense of category theory may be proved by more or less obvious manipulations within the algebraic structure. The uniqueness statement theorem now follows from general principles, see [18, Corollary 1, p. 83]. This completes the proof.

REMARK 5.3. In our definition of the induced and co-induced representations we might have started with a different set of actions on $E$ and $E^{\prime}$. Indeed, if we invert all the actions (K1), (K2), (G1) and (G2) and proceed as before with obvious modifications on the way, we obtain induced and co-induced representations ${ }^{G} \pi^{\prime}$ and ' $\pi^{G}$ that are seemingly different from ${ }^{G} \pi$ and $\pi^{G}$ respectively. However, the Frobenius reciprocity theorem will also hold for these (or their associated left or right $G$-modules). It therefore follows from the uniqueness statement of the theorem that ${ }^{G} \pi^{\prime}={ }^{G} \pi$ and ${ }^{\prime} \pi^{G}=\pi^{G}$.

6. Realizations. In this section we give alternative descriptions of the $G$-modules ${ }^{G} E$ and $E^{G}$. We preserve the preceding notation and assumptions. As may be expected $E^{G}$ may be realized as a space of $E$-valued $C^{\infty}$-functions on $G$, whereas such a realization for ${ }^{G} E$ in general is impossible. Since the realization of $E^{G}$ is by far the simplest to achieve, we treat it first.

Let $E_{K}(G, E)$ denote the space of $C^{\infty}$-functions $f: G \rightarrow E$ which satisfies

$$
f(x k)=\pi\left(k^{-1}\right) f(x) \text {. }
$$

$E_{K}(G, E)$ is a closed subspace of $E(G, E)$ and equipped with the relative topology it becomes a complete locally convex space. We have a natural right action of $G$ on $E(G, E)$ which leaves $E_{K}(G, E)$ invariant, viz:

$$
(f x)(y)=f(x y) \quad(x, y \in G) .
$$

The associated representation ' $\pi^{G}$ of $G$ on $E_{K}(G, E)$ is then given by

$$
\left[\pi^{G}(x) f\right](y)=f\left(x^{-1} y\right) \text {. }
$$

Proposition 6.1. $E^{G}$ and $E_{K}(G, E)$ are equivalent differentiable right G-modules, i.e. $\pi^{G} \cong{ }^{\prime} \pi^{G}$.

Proof. Let $\Gamma: E(G, E) \rightarrow L\left(E^{\prime}, E\right)$ be as in $\S 3$, i.e. $\Gamma(f)(S)=S^{\prime} f$, so $\Gamma$ 
is an isomorphism onto. All we have to do is to show that $\Gamma$ maps $E_{K}(G, E)$ onto $L_{K}\left(E^{\prime}, E\right)$ and intertwines the action of $G$. If $f \in E(G, E)$ and satisfies (6.1) then

$$
\begin{aligned}
\Gamma(f)(S k) & =\Gamma(f)\left(S * \epsilon_{k}\right)=\left(\Gamma(f) \circ \check{\epsilon}_{k}\right)(S)=\Gamma\left(f * \check{\epsilon}_{k}\right)(S)=\Gamma(k f)(S) \\
& =\Gamma\left(\pi\left(k^{-1}\right) f\right)(S)=S^{\prime}\left(\pi\left(k^{-1}\right) \circ f\right)=\pi\left(k^{-1}\right)\left(S^{\prime} f\right)=\left(S^{\prime} f\right) k=(\Gamma(f) S) k
\end{aligned}
$$

where we have used successively (4.2), (3.5), (3.7), (4.1), (6.1) and (3.8). Hence $\Gamma(f)$ is $K$-linear and belongs to $L_{K}\left(E^{\prime}, E\right)$. The converse is similar. Next, if $x \in G$ :

$$
\Gamma(f x)(S)=S^{\prime}(f x)=(x S)^{\prime} f=\Gamma(f)(x S)=(\Gamma(f) x)(S)
$$

for all $S \in E^{\prime}$. Hence $\Gamma(f x)=\Gamma(f) x$ and the proof is complete.

We will now give a realization of ${ }^{G} E$ which is valid in important special cases, notably if $E$ is the strong dual of a reflexive Fréchet space. This realization of the $K$-tensor product $E^{\prime} \bar{\otimes}_{K} E$ is at least to the author's knowledge new, and appears to be of some interest in itself.

Let $E_{K}^{\prime}(G, E)$ denote the space of $E$-valued distributions $m$ on $G$ satisfying the following two conditions:

supp $m \subseteq C K$ for some compact subset $C$ of $G$

(i.e. $m$ has compact support modulo $K$ ),

$$
m(k \varphi)=\delta_{K}\left(k^{-1}\right) k m(\varphi) \quad\left(k \in K, \varphi \in D(G), \delta_{K}=\right.\text { modular function }
$$

We topologize $E_{K}^{\prime}(G, E)$ as follows. For each compact set $C \subseteq G$ let $E_{C, K}^{\prime}(G, E)$ be the subspace of those $m$ 's that have their support in $C K$. We give this space the relative topology as a closed subspace of $D^{\prime}(G, E)$, and where the latter is given the usual topology of uniform convergence on bounded (i.e. compact) subsets of $D(G) . E_{K}^{\prime}(G, E)$ is then given the inductive limit topology from the family of spaces $E_{C, K}^{\prime}(G, E)$ as $C$ runs through the collection of compact subsets of G. $G$ is countable at infinity so $E_{K}^{\prime}(G, E)$ is a strict inductive limit of complete spaces and is therefore itself complete [12]. $E_{K}^{\prime}(G, E)$ is made into a differentiable left $G$-module by the action

$$
(x m)(\varphi)=m(\varphi x) \quad(x \in G, \varphi \in D(G)) .
$$

PROPOSITION 6.2. If $E$ is a normed space or the strong dual of a reflexive Fréchet space then ${ }^{G} E$ and $E_{K}^{\prime}(G, E)$ are equivalent differentiable left $G$-modules.

The proof of this result will be given through a sequence of lemmas in the next section. Proposition 6.2 is false without some assumption on $E$. For instance it fails if $E=E$. For a discussion see the end of $\$ 7$.

7. Proof of Proposition 6.2. As before $G$ is a Lie group, countable at infinity and $\pi$ is a differentiable representation of the closed subgroup $K$ on a complete locally convex space $E$. 
Let $E^{\prime}(G, E)$ denote the space of $E$-valued distributions with compact support. We equip this space with the inductive limit topology described in the preceding section (taking $K=\{e\}$ ). We obtain a right action of $K$ of this space by

$$
(m k)(\varphi)=\delta_{K}(k) k^{-1} m(k \varphi) .
$$

Then define

$$
m^{\#} \varphi=\int_{K}(m k)(\varphi) d k
$$

where integration is with respect to right Haar-measure on $K$. The integral defines an element of $E$ since the map $k \rightarrow(m k)(\varphi)$ is easily seen to be continuous of $K$ into $E$ and has compact support, for fixed $m$ and $\varphi$. In fact, if supp $m \subseteq C_{1}$ and supp $\varphi \subseteq C_{2}$ then $\operatorname{supp}(k \rightarrow(m k)(\varphi)) \subseteq\left\{k \in K: C_{1} k \cap C_{2} \neq \varnothing\right\}$ since supp $m k \subseteq C_{1} k$. Hence $x k=y$ for some $x \in C_{1}, y \in C_{2}$ or $k=x^{-1} y \in C_{1}^{-1} C_{2}$. So $\operatorname{supp}(k \rightarrow(m k)(\varphi)) \subseteq K \cap C_{1}^{-1} C_{2}$.

The map $\varphi \rightarrow m^{\#} \varphi$ is evidently linear of $D(G)$ into $E$.

Lemma 7.1. \#: $m \rightarrow m^{\#}$ is a linear, continuous and open map of $E^{\prime}(G, E)$ onto $E_{K}^{\prime}(G, E)$.

Proof. Linearity of \# is obvious. Clearly, if $m$ has support in $C$, then supp $m^{\#} \subseteq C K$. Let $m \in E^{\prime}(G, E)$ and suppose supp $m \subseteq C_{1}$. We must show that $m^{\#}: D(G) \rightarrow E$ is continuous. Let $V$ be a neighbourhood of 0 in $E$. Let $C_{2}$ be an arbitrary compact subset of $G$, and let $\mu$ be right Haar measure on $K$. If $\mu\left(K \cap C_{1}^{-1} C_{2}\right) \neq 0$ put $r=\mu\left(K \cap C_{1}^{-1} C_{2}\right)^{-1}$. Since $\pi$ is jointly continuous there is a neighbourhood $V_{1}$ of 0 in $E$ such that $\pi\left(k^{-1}\right) V_{1} \subseteq r V$ for all $k \in K \cap$ $C_{1}^{-1} C_{2} . m$ is continuous, so there is a neighbourhood $U$ of 0 in $D(G)$ such that $m(U) \subseteq V_{1}$. Finally, the action of $K$ on $D(G)$ is jointly continuous so there is a neighbourhood $U_{1}$ of 0 in $D(G)$ such that $\delta_{K}(k) k \varphi \in U$ for all $k \in K \cap C_{1}^{-1} C_{2}$ and all $\varphi \in U_{1}$. Hence, if $\varphi \in U_{1}$ then

$$
\delta_{K}(k) k^{-1} m(k \varphi) \subseteq k^{-1} m(U) \subseteq k^{-1} V_{1} \subseteq r V
$$

for all $k \in K \cap C_{1}^{-1} C_{2}$. Consequently, if $a^{\prime} \in V^{0}$ and supp $\varphi \subseteq C_{2}$ we get

$$
\left\langle m^{\#} \varphi, a^{\prime}\right\rangle|=| \int_{K \cap c_{1}^{-1} c_{2}}\left\langle\delta_{K}(k) k^{-1} m(k \varphi), a^{\prime}\right\rangle d k \mid \leqslant \int_{K \cap c_{1}^{-1} c_{2}} r d k=1
$$

which shows that $m^{\#} \varphi \in\left(V^{0}\right)_{0}$. This shows that $m^{\#}$ is continuous on $D_{C_{2}}(G)$. Since $C_{2}$ was arbitrary and $D(G)$ is given the inductive limit topology, it follows that $m^{\#}: D(G) \rightarrow E$ is continuous. Now let $k_{1} \in K$ be fixed and let $\varphi \in D(G)$. Then we get 


$$
\begin{aligned}
& \left(m^{\#} k_{1}\right)(\varphi)=\delta_{K}\left(k_{1}\right) k_{1}^{-1} m^{\#}\left(k_{1} \varphi\right)=\delta_{K}\left(k_{1}\right) k_{1}^{-1} \int_{K} \delta_{K}(k) k^{-1} m\left(k k_{1} \varphi\right) d k \\
& \quad=\int_{K} \delta_{K}\left(k k_{1}\right) k_{1}^{-1} k^{-1} m\left(k k_{1} \varphi\right) d k=\int_{K}\left(m k k_{1}\right)(\varphi) d k=\int_{K}(m k)(\varphi) d k=m^{\#}(\varphi)
\end{aligned}
$$

Hence $m^{\#} k_{1}=m^{\#}$ so $m^{\#} \in E_{K}^{\prime}(G, E)$.

Next we show that $m \rightarrow m^{\#}$ is continuous. Since $E^{\prime}(G, E)$ is given the inductive limit topology it suffices to show that \# restricted to $E_{C}^{\prime}(G, E)$ is continuous for an arbitrary compact $C \subseteq G$. Let $W$ be a neighbourhood of 0 in $E_{K}^{\prime}(G, E)$. Then $W \cap E_{C, K}^{\prime}(G, E)$ is a 0 -neighbourhood in the relative topology from $D^{\prime}(G, E)$ and therefore contains one of the form $W_{B, V} ; B$ bounded in $D(G), V$ a 0 -neighbourhood in $E$. \# maps $E_{C}^{\prime}(G, E)$ into $E_{C, K}^{\prime}(G, E)$ so it suffices to find a neighbourhood $W_{B_{1}}, V_{1}$ of 0 in $D^{\prime}(G, E)$ such that \#: $W_{B_{1}}, V_{1} \cap$ $E_{C}^{\prime}(G, E) \rightarrow W_{B, V} \cap E_{C, K}^{\prime}(G, E)$. Now, since $B$ is bounded in $D(G)$ it is contained in $D_{C_{1}}(G)$ for some compact set $C_{1} \subseteq G$. The action of $K$ on $D(G)$ is jointly continuous so the set $B_{1}=\left(K \cap C^{-1} C_{1}\right) B$ is relatively compact in $D(G)$. By the joint continuity of $\pi$ there is a neighbourhood $V_{1}$ of 0 in $E$ such that $\delta_{K}(k) k^{-1} V_{1} \subseteq V$ for all $k \in K \cap C^{-1} C_{1}$. Let $V_{2}=r V_{1}, r=\mu\left(K \cap C^{-1} C_{1}\right)^{-1}$. Suppose now that $m \in W_{B_{1}, V_{2}} \cap E_{C}^{\prime}(G, E)$ and that $\varphi \in B$ (so supp $\varphi \subseteq C_{1}$ ), $a^{\prime} \in V^{0}$ :

$$
\left.K m^{\#} \varphi, a^{\prime}\right\rangle|=| \int_{K \cap c^{-1} C_{1}}\left\langle\delta_{K}(k) k^{-1} m(k \varphi), a^{\prime}\right\rangle d k \mid \leqslant 1
$$

so $m^{\#} \in W_{B, V} \cap E_{C, K}^{\prime}(G, E) \subseteq W$ and continuity of \# is proved. Let $m \in$ $D^{\prime}(G, E), h \in \mathcal{D}(G)$. We define

$$
(h m)(\varphi)=m(h \varphi) \quad(\varphi \in D(G)) .
$$

Since $\varphi \rightarrow h \varphi$ is a continuous linear map of $D(G)$ into itself, $h m$ belongs to $E^{\prime}(G, E)$. To show that \# is surjective let $m \in E_{K}^{\prime}(G, E)$ with supp $m \subseteq C K$. Let $h \in D(G), \varphi \in D(G)$ :

$$
\begin{aligned}
(h m)^{\#}(\varphi) & =\int_{K}(h m k)(\varphi) d k=\int_{K} \delta_{K}(k) k^{-1} m(h(k \varphi)) d k \\
& =\int_{K} \delta_{K}(k) k^{-1} m\left(k\left(k^{-1} h \varphi\right)\right) d k \\
& =\int_{K}(m k)\left(k^{-1} h \varphi\right) d k=\int_{K} m\left(k^{-1} h \varphi\right) d k .
\end{aligned}
$$

Now $k \rightarrow k^{-1} h$ is continuous from $K$ into $D(G)$, hence also from $K$ into $E(G)$. $E(G)$ is reflexive and its dual consists of the distributions of compact support, so the integral $\int_{K} k^{-1} h d k$ exists as an element $h^{0}$ of $E(G)$. We clearly have

$$
h^{0}(x)=\int_{K} h\left(x k^{-1}\right) d k \text {. }
$$


Now $\varphi m$ belongs to $E^{\prime}(G, E)$ and therefore extends to a continuous linear map of $E(G)$ into $E$. Hence

$$
\int_{K} m\left(k^{-1} h \cdot \varphi\right) d k=\int_{K}(\varphi m)\left(k^{-1} h\right) d k=(\varphi m)\left(h^{0}\right)=m\left(\varphi h^{0}\right) .
$$

We have therefore shown that

$$
(h m)^{\#}(\varphi)=m\left(\varphi h^{0}\right) \quad(\varphi \in D(G)) .
$$

At this stage we need the following well-known result [10].

LEMMA 7.2. For each $h \in D(G)$ the function $h^{0}$ defined by (7.4) belongs to $E(G)$ and is constant on left cosets $x K$. We have supp $h^{0} \subseteq(\operatorname{supp} h) K$. The function $h^{00}(x K)=h^{0}(x)$ has compact support and belongs to $D(G / K)$. If $g \in$ $D(G / K)$ there is a function $h \in \mathcal{O}(G)$ such that $g=h^{00}$. If $g \geqslant 0$ we may take $\boldsymbol{h} \geqslant 0$.

In particular, on the basis of the lemma we may choose $h \in D(G)$ such that $h^{0} \equiv 1$ on $C_{1} K$ where $C_{1}$ is a compact neighbourhood of $C(C K=\operatorname{supp} m)$. Then for any $\varphi \in D(G), \varphi-h^{0} \varphi$ vanishes identically on a neighbourhood of $C K$ so by (7.5)

$$
m(\varphi)=m\left(\varphi h^{0}\right)=(h m)^{\#}(\varphi)
$$

Hence $m=(h m)^{\#}$ and \# is surjective.

It remains to show that \# is open. Let $W$ be a neighbourhood of 0 in $E^{\prime}(G, E)$. It suffices to show that $W^{\#} \cap E_{C, K}^{\prime}(G, E)$ is a neighbourhood of 0 for an arbitrary compact $C \subseteq G$. So let $C$ be given and take $h \in D(G)$ as above with $h^{0} \equiv 1$ on $C_{1} K, C_{1} \supseteq C$. Cearly supp $h=C_{2} \supseteq C_{1}$. We claim that the map $\rho: m \rightarrow h m$ of $E_{C, K}^{\prime}(G, E)$ into $E_{C_{2}}^{\prime}(G, E)$ is continuous. Indeed, let $W_{B, V}$ be a neighbourhood of 0 in $E_{C_{2}}^{\prime}(G, E) . \varphi \rightarrow h \varphi$ is continuous of $D(G)$ into itself so $B_{1}=\{h \varphi: \varphi \in B\}$ is bounded in $D(G)$. Hence, if $m \in W_{B_{1}, V}$ then $(h m)(\varphi)=$ $m(h \varphi) \in V$ for all $\varphi \in B$ so $h m \in W_{B, V}$. Hence $\rho$ is continuous and $\rho^{-1}\left(W \cap \rho\left(E_{C, K}^{\prime}(G, E)\right)\right.$ is a 0-neighbourhood in $E_{C, K}^{\prime}(G, E)$. Now $\rho$ is injective on $E_{C, K}^{\prime}(G, E)$, in fact $(h m)^{\#}=m$ so $\rho^{-1}=\#$ by (7.6). Consequently $\rho^{-1}\left(W \cap \rho\left(E_{C, K}^{\prime}(G, E)\right)\right)=\left(W \cap \rho\left(E_{C, K}^{\prime}(G, E)\right)\right)^{\#} \subseteq W^{\#} \cap E_{C, K}^{\prime}(G, E)$ and the proof is complete.

Let $S \in E^{\prime}(G)$ and $a \in E$ be arbitrary. We define $m_{S, a}(\varphi)=S(\varphi) a \quad(\varphi \in$ $D(G)$ ). Clearly $m_{S, a}$ belongs to $E^{\prime}(G, E)$.

Lemma 7.3. Let $k_{1} \in K$ be fixed. Then

$$
m_{S k_{1}, a}^{\#}=m_{S, k_{1} a}^{\#}
$$


Proof. If $\varphi \in D(G)$ we have

$$
\begin{aligned}
m_{S k_{1}, a}^{\#}(\varphi) & =\int_{K} \delta_{K}(k) k^{-1} m_{S k_{1}, a}(k \varphi) d k=\int_{K^{\prime}} \delta_{K}(k)\left(S k_{1}\right)(k \varphi) k^{-1} a d k \\
& =\int_{K} \delta_{K}(k) S\left(k_{1} k \varphi\right) k^{-1} a d k \\
& =\int_{K} \delta_{K}\left(k^{-1}\right) \delta_{K}(l) S(l \varphi) l^{-1} k_{1} a d\left(k_{1}^{-1} l\right) \quad\left(l=k_{1} k\right) \\
& =\int_{K} \delta_{K}(l) S(l \varphi) l^{-1} k_{1} a d l=m_{S, k_{1} a}^{\#}(\varphi)
\end{aligned}
$$

which proves the assertion.

REMARK 7.4. This is the first instance where the modular function $\delta_{K}$ in the definition of \# plays a role.

Lemma 7.5. The bilinear map $(S, a) \rightarrow m_{S, a}$ of $E^{\prime} \times E$ into $E^{\prime}(G, E)$ is hypocontinuous.

Proof. (1) Let $B_{1} \subseteq E^{\prime}$ be bounded, and let the neighbourhood $W=$ $W_{B, V}$ of 0 in $E^{\prime}(G, E)$ be given. (By the next lemma $B_{1} \subseteq E_{C}^{\prime}(G, E)$ for some compact $C \subseteq G$ so it is permissible to assume that $W$ is of this form.) $B$ is bounded in $D(G)$ and the injection of $D(G)$ into $E(G)$ is continuous, so $B$ is bounded as a subset of $E(G)$. Hence $B^{0}$ is a 0 -neighbourhood in $E^{\prime}$ and there is a real number $r>0$ such that $B_{1} \subseteq r B^{0}$. Then, if $U=V / r$ and $a \in U$ we have $m_{S, a}(\varphi)=S(\varphi) a \in V$, for all $S \in B_{1}$ and all $\varphi \in B$. Hence $m_{S, a} \in W_{B, V}$ for $S \in B_{1}, a \in U$.

(2) Let $A \subseteq E$ be bounded. We must show that the family of linear maps $\left\{S \rightarrow m_{S, a}: a \in A\right\}$ is equicontinuous. Since $E^{\prime}$ is barreled it suffices to show that $S \rightarrow m_{S, a}$ is continuous for fixed $a \in E$, and that $\left\{m_{S, a}: a \in A\right\}$ is bounded in $E^{\prime}(G, E)$ for each $S \in E^{\prime}$. This last statement follows from (1) since for fixed $S$ the map $a \rightarrow m_{S, a}$ is continuous and maps bounded sets into bounded sets. Now let $a \in E$ be fixed. Since $E^{\prime}$ is an inductive limit of $\left\{E_{C}^{\prime}: C\right.$ compact in $\left.G\right\}$ (next lemma) it suffices to show that $S \rightarrow m_{S, a}$ is continuous on $E_{C}^{\prime}$ for arbitrary $C$. But then $m_{S, a}$ has support in $C$, so we may take a neighbourhood of 0 of the type $W_{B, V}$ in $E^{\prime}(G, E)$. Then $A \subseteq r V$ for some $r>0$ so if $S \in B^{0} / r$ then $m_{S, a} \in W_{B, V}$ for all $a \in A$. This completes the proof.

LEMMA 7.6. The strong topology of $E^{\prime}$ as a dual of $E$ coincides with the inductive limit topology of $E^{\prime}(G, \mathrm{C})$.

Proof. It is well known that $E^{\prime}$ may be identified with the distributions with compact support, i.e. with $E^{\prime}(G, C)$ as a vector space. To show that the topologies are the same let $i: E^{\prime}(G, \mathrm{C}) \rightarrow E_{b}^{\prime}$ denote the identity map. We first show that $i$ is continuous. Fix the compact set $C \subseteq G$. Let $W_{B, e}$ be an arbitrary 
neighbourhood of 0 in $E_{b}^{\prime} ; B$ a bounded subset of $E, \epsilon>0$. Choose $h \in D(G)$ such that $h \equiv 1$ on a neighbourhood of $C$. Then $h B$ is a bounded subset of $D(G)$. Indeed, if $T \in D^{\prime}$ then $T(h B)=(h T)(B)$ and $h T \in E^{\prime}$. Hence $h B$ is weakly bounded and therefore bounded in $D$. Consequently, if $S$ belongs to $W_{h B, \epsilon} \cap E_{C}^{\prime}(G, \mathbf{C})$ then for $\varphi \in B:|S(\varphi)|=|S(h \varphi)|<\epsilon$ which shows that $i(S) \in W_{B, \epsilon}$. So $i \mid E_{C}^{\prime}(G, \mathbf{C})$ is continuous. Since $E^{\prime}(G, C)$ is an inductive limit $i$ is continuous. Now $E_{b}^{\prime}$ is a Mackey space so its topology is the strongest locally convex topology compatible with its topological dual $E$. Hence, to prove that the two topologies in question are the same it now suffices to show that each continuous linear functional $\varphi$ on $E^{\prime}(G, C)$ is also continuous on $E_{b}^{\prime}$. So let such a $\varphi$ be given. To show that $\varphi$ is continuous on $E_{b}^{\prime}$ it suffices to show that $\varphi$ is $w^{*}$-continuous. Now $E$ is complete so this will follow if we can show that $\varphi \mid A$ is $w^{*}$-continuous for every equicontinuous subset $A$ of $E_{b}^{\prime}$ (Grothendieck's completeness theorem [12, 16.9]). Let $A$ be a $w^{*}$-closed equicontinuous subset of $E_{b}^{\prime} . A$ is bounded in $E_{b}^{\prime}$, hence compact since $E_{b}^{\prime}$ is a nuclear space. We claim that all $S \in A$ must have their support inside a fixed compact $C \subseteq G$. If not, let $\bigcup_{n} C_{n}=G ; C_{n}$ compact, $n=1,2$, .... For each $n$ there must be an $S_{n} \in A$ and a $\varphi_{n} \in D(G)$ supported by a small neighbourhood $U_{n}$ such that $U_{n} \cap C_{n}=\varnothing$, and $S_{n}\left(\varphi_{n}\right)=1$. Clearly $\varphi_{n} \rightarrow$ 0 in $E$. But then, by reflexivity $\varphi_{n}(S)=S\left(\varphi_{n}\right) \rightarrow 0$ uniformly for $S \in A$, contradicting $S_{n}\left(\varphi_{n}\right)=1$. Hence $A \subseteq E_{C}^{\prime}(G, \mathrm{C})$ for some compact $C \subseteq G$. But clearly the relative topology from $D^{\prime}$ and the relative topology from $E_{b}^{\prime}$ coincide on $E_{C}^{\prime}(G, C)$ since the injection $E_{b}^{\prime} \rightarrow D^{\prime}$ is continuous, and we already know that $i$ is continuous. So $\varphi \mid A$ is strongly continuous, hence $w^{*}$-continuous since the strong and the $w^{*}$-topologies coincide on $A$ by compactness. The proof is complete.

We are now in a position to define the isomorphism $E^{\prime} \bar{\otimes}_{K} E \rightarrow E_{K}^{\prime}(G, E)$ which is the object of this section. Let $\omega$ be the bilinear map $(S, a) \rightarrow m_{S, a}^{\#}$ of $E^{\prime}$ $\times E$ into $E_{K}^{\prime}(G, E)$. By the preceding results $\omega$ is hypocontinuous and $K$-balanced. So $\omega$ determines a continuous linear map $\Omega: E^{\prime} \otimes_{K} E \rightarrow E_{K}^{\prime}(G, E)$ such that $\Omega(S \otimes a)=m_{S, a}^{\#}$. Our first objective is to show that $\Omega$ is injective. For this some further preparation is necessary. Since $E$ is a (differentiable) left $K$-module we realize $E^{\prime}$ as a right $K$-module in the usual way: $\left\langle k a, a^{\prime}\right\rangle=\left\langle a, a^{\prime} k\right\rangle(a \in E$, $\left.a^{\prime} \in E^{\prime}, k \in K\right)$. We do not assume that $E^{\prime}$ is complete, but we still have $E_{b}^{\prime}=\breve{E}$. Suppose that $f: G \rightarrow E_{b}^{\prime}$ is differentiable and satisfies (see (6.1))

$$
f(x k)=f(x) k \quad(x \in G, k \in K) .
$$

Let $E_{K}^{\epsilon}(G, \check{E})$ denote the subspace of $E_{K}(G, \check{E})$ consisting of functions $f$ satisfying the following property:

$\left(\mathrm{P}_{\epsilon}\right)$ For each compact set $C \subseteq G$ and each $D \in \mathscr{D}(\mathbb{C}), D f(C)$ is an equicontinuous subset of $\check{E}$.

(Observe that if $f \in E_{K}(G, \breve{E})$ then $D f \in E_{K}(G, \check{E})$ by an easy computation.) 
Now let $F$ be a continuous linear functional on $E^{\prime} \otimes_{K} E$ (equipped with the inductive topology $\beta$ ) and define

$$
\langle a, f(x)\rangle=F\left(\epsilon_{x} \otimes a\right) \quad(x \in G, a \in E) .
$$

Lemma 7.7. The map $F \rightarrow f$ defined by (7.8) is a linear isomorphism of $\left(E^{\prime} \otimes_{K} E\right)^{\prime}$ onto $E_{K}^{\epsilon}(G, \check{E})$. The inverse map is given by

$$
F(S \otimes a)=\int_{G}\langle a, f(x)\rangle d S(x) .
$$

Moreover the map is a topological isomorphism with respect to the topology of bi-bounded convergence on the dual of $E^{\prime} \otimes_{K} E$ and the relative topology on $E_{K}^{\epsilon}(G, \breve{E})$ as a subspace of $E\left(G, E_{b}^{\prime}\right)$.

Proof. Let $f \in E_{K}^{\epsilon}(G, \check{E})$ be fixed and define $\omega_{f}(S, a)=s\langle a, f(x)\rangle d S(x)$ $=S(a \circ f), \omega_{f}$ is bilinear and $K$-balanced: $\omega_{f}(S k, a)=S k(a \circ f)=S(k(a \circ f))=$ $S(k a \circ f)$ (since $f$ satisfies (7.7)) $=\omega_{f}(S, k a)$. Hence $\omega_{f}$ determines a linear functional $F$ on $E^{\prime} \otimes_{K} E$ which satisfies (7.9). We must show that $F$ is continuous, or equivalently, that $\omega_{f}$ is hypocontinuous.

(1) Suppose $A \subseteq E^{\prime}$ is bounded. Then $A$ is equicontinuous so there is a continuous seminorm $p_{D, C}$ on $E(D \in \mathfrak{D}(\mathbb{S}), C$ compact $\subseteq G)$ such that $p_{D, C}(\varphi)$ $=\sup _{x \in C}|D \varphi(x)|<1=|S(\varphi)|<1$ for all $S \in A$. Now by assumption $D f(C)$ is an equicontinuous subset of $\breve{E}$, so there is a neighbourhood $V$ of 0 in $E$ such that $D f(C) \subseteq V^{0}$. Hence

$$
p_{D, C}(a \circ f)=\sup _{x \in C}|D(a \circ f)(x)|=\sup _{x \in C}|\langle a, D f(x)\rangle| \leqslant 1 .
$$

Consequently $\left|\omega_{f}(S, a)\right|=|S(a \circ f)| \leqslant 1$ for all $S \in A$ and all $a \in V$.

(2) Suppose $B \subseteq E$ is bounded. We must show that the family of maps $S \rightarrow \omega_{f}(S, a)=S(a \circ f) \quad(a \in B)$ is equicontinuous. Again, as in the proof of Lemma 7.5 it suffices by (1) to show that $S \rightarrow S(a \circ f)$ is continuous for fixed $a \in E$. But this is trivial since $a \circ f \in E$.

Hence $\omega_{f}$ is hypocontinuous so $F$ is continuous. The map $\theta: f \rightarrow F$ of $E_{K}^{\epsilon}(G, \breve{E})$ into the dual of $E^{\prime} \otimes_{K} E$ is clearly linear and injective. In fact, if $F=0$ then $0=F\left(\epsilon_{x} \otimes a\right)=\langle a, f(x)\rangle$ for all $a \in E$, so $f(x)=0$.

To show that $\theta$ is surjective, let $F \in\left(E^{\prime} \otimes_{K} E\right)^{\prime}$ and define $f$ by (7.8). We claim that if $D \in D(\mathbb{D})$ th $~ m$

$$
F\left(D^{\#} \epsilon_{x} \otimes a\right)=\langle a, D f(x)\rangle \quad(x \in G, a \in E)
$$

which will imply that $f$ is differentiable. To prove (7.10) let $X \in \mathbb{B}$. Then

$$
\begin{aligned}
F\left((-X) \epsilon_{x} \otimes a\right) & =\lim _{t \rightarrow 0} t^{-1} F\left(\left(\epsilon_{\exp t X} * \epsilon_{x}-\epsilon_{x}\right) \otimes a\right) \\
& =\lim _{t \rightarrow 0}\left\langle a, t^{-1}\{f(\exp t X x)-f(x)\}\right\rangle=\langle a, X f(x)\rangle
\end{aligned}
$$


which establishes $X f(x)$ as a linear functional on $E$. Since $a \rightarrow F\left((-X) \epsilon_{x} \otimes a\right)$ is continuous $X f(x) \in E^{\prime}$. (7.10) now follows by induction. Hence $f \in E\left(G, E_{\sigma}^{\prime}\right)$. That $f$ actually belongs to $E\left(G, E_{b}^{\prime}\right)$, i.e. that $D f(x)$ exists in the strong topology of $E^{\prime}$ follows directly from the hypocontinuity of the canonical map $(S, a) \rightarrow$ $S \otimes a$. Now let $k \in K$. Then

$$
\langle a, f(x k)\rangle=F\left(\epsilon_{x k} \otimes a\right)=F\left(\epsilon_{x} k \otimes a\right)=F\left(\epsilon_{x} \otimes k a\right)=\langle k a, f(x)\rangle=\langle a, f(x) k\rangle
$$

for all $a \in E$. Hence $f$ satisfies (7.7) and belongs to $E_{K}(G, \check{E})$. It remains to see that $f$ has the property $\left(\mathrm{P}_{\epsilon}\right)$. So let $D \in \mathscr{P}(\mathbb{E})$ and let $C \subseteq G$ be compact. The map $x \rightarrow \epsilon_{x}$ is continuous of $G$ into $E^{\prime}$ and $D^{\#}$ is continuous on $E^{\prime}$, so $\left\{D^{\#} \epsilon_{x}: x \in C\right\}$ is compact in $E^{\prime}$. Hence there is a neighbourhood $V$ of 0 in $E$ such that $K a, D f(x)\rangle|=| F\left(D^{\#} \epsilon_{x} \otimes a\right) \mid \leqslant 1$ for all $x \in C$ and all $a \in V$. Hence $f$ satisfies $\left(\mathrm{P}_{\epsilon}\right)$. This completes the first part of the proof. The topological part is now a simple verification and is left to the reader. The proof is complete.

ReMARK 7.8. Under additional assumptions on $E$ the conclusion of Lemma 7.7 may be sharpened considerably. For instance if $E$ is a Mackey space so that each $w^{*}$-compact convex subset of $E^{\prime}$ is equicontinuous the property $\left(\mathrm{P}_{\epsilon}\right)$ is automatic. This discussion will be completed in the next section when we take up the problem of representing the dual modules of ${ }^{G} E$ and $E^{G}$.

We now return to the proof of Proposition 6.2. Our first aim is to produce a certain separating family of continuous linear functionals on $E^{\prime} \otimes_{K} E$. We still do not assume that $E_{b}^{\prime}$ is complete and have to pay for this by facing some technicalities.

Let $F$ be a right differentiable $K$-module, $F$ a complete locally convex space. Let $D_{K}(G, F)$ denote the space of differentiable functions $f: G \rightarrow F$ satisfying the following two conditions.

$$
\text { supp } f \subseteq C K \text { for some compact subset } C \subseteq G \text {, }
$$

$$
f(x k)=f(x) k \text {. }
$$

We topologize $D_{K}(G, F)$ as follows: For each compact $C \subseteq G$ let $D_{C, K}(G, F)$ denote the subspace of those $f$ 's that have their support in $C K$. Give this space the relative topology from $E(G, F)$. It is clearly closed, hence complete in the relative topology. We give $D_{K}(G, F)$ the inductive limit topology. It is a strict inductive limit, hence complete. If $F$ is a Fréchet space it is of the type $L F$ [2].

For $f \in E(G, F)$ put

$$
(k f)(x)=\delta_{K}(k) f(x k) k^{-1} \quad(x \in G, k \in K)
$$

and define, if $f$ has compact support:

$$
\widetilde{f}(x)=\int_{K}(k f)(x) d k .
$$


It is easily seen that $f$ is a differentiable function of $G$ into $F$, and has support in (supp $f$ ) $K$. Moreover, if $k_{1} \in K$ :

$$
\begin{aligned}
\vec{f}\left(x k_{1}\right) & =\int_{K}(k f)\left(x k_{1}\right) d k=\int_{K} \delta_{K}(k) f\left(x k_{1} k\right) k^{-1} d k \\
& =\int_{K} \delta_{K}\left(k_{1}^{-1}\right) \delta_{K}(l) f(x l) l^{-1} k_{1} d\left(k^{-1} l\right) \quad\left(k_{1} k=l\right) \\
& =\int_{K} \delta_{K}(l) f(x l) l^{-1} k_{1} d l=\vec{f}(x) k_{1}
\end{aligned}
$$

so $f$ belongs to $D_{K}(G, F)$.

LEMMA 7.9. $f \rightarrow T$ is a linear, continuous and open map of $D(G, F)$ onto $D_{K}(G, F)$.

Proof. This is (essentially) Bruhat's Proposition 4.1. [2].

Now let $F$ be the completion of $\breve{E}$. Then $F$ is a right differentiable $K$-module, and the results above apply. Let $\mathcal{D}^{\epsilon}(G, \check{E})$ (resp. $\mathcal{D}_{K}^{\epsilon}(G, \check{E})$ ) denote the subspace of $\mathcal{O}(G, \check{E})$ (resp. $D_{K}(G, \check{E})$ ) consisting of functions satisfying $\left(\mathrm{P}_{\epsilon}\right)$.

Lemma 7.10. $\neg$ maps $D^{\epsilon}(G, \check{E})$ into $D_{K}^{\epsilon}(G, \check{E})$.

Proof. Let $D \in \mathscr{D (}(\mathbb{S})$, clearly $D \dot{f}=\overparen{D f}$ since $D$ is right invariant. Now let $f \in D^{\epsilon}(G, \breve{E})$ and let $C \subseteq G$ be compact. If supp $f=C_{1}$ then $k \rightarrow D f(x k)$ has support in $C^{-1} C_{1} \cap K=C_{2}$ which is a compact subset of $K$. Let $r=\mu\left(C_{2}\right)^{-1}$. By assumption $D f\left(C_{1}\right)$ is equicontinuous in $\breve{E}$, so there is a neighbourhood $U$ of 0 in $E$ such that $\left\langle U, D f\left(C_{1}\right)\right\rangle \mid \leqslant r$. Now take the neighbourhood $V$ of 0 in $E$ such that $\delta_{K}(k) k a \in U$ for all $k \in C_{2}$ and all $a \in V$. Then if $x \in C$ :

$$
\left.K a, D \vec{f}(x)\rangle|=| \int_{C_{2}} \delta_{K}(k) \backslash k a, D f(x k)\right\rangle d k \mid \leqslant \int_{C_{2}} r d k=1
$$

for all $a \in V$. Hence $D \vec{f}(C)$ is equicontinuous. In particular $\bar{f}$ maps $G$ into $E^{\prime}$, hence into $\check{E}$. The proof is complete.

Let $\varphi \in D(G)$ and let $a^{\prime} \in \check{E}$. Consider the bilinear form $\omega_{\varphi, a^{\prime}}$ on $E^{\prime} \times E$ given by

$$
\omega_{\varphi, a^{\prime}}(S, a)=\left\langle m_{S, a}^{\#}(\varphi), a^{\prime}\right\rangle .
$$

It is clearly $K$-balanced and hypocontinuous, so it determines a continuous linear functional $F_{\varphi, a}$, on $E^{\prime} \otimes_{K} E$ such that

$$
F_{\varphi, a^{\prime}}(S \otimes a)=\left\langle m_{S, a}^{\#}(\varphi), a^{\prime}\right\rangle .
$$

Let $F$ be the family $\left\{F_{\varphi, a^{\prime}}: \varphi \in D, a^{\prime} \in \check{E}\right\}$.

LEMMA 7.11. F spans a dense linear subspace of $\left(E^{\prime} \otimes_{K} E\right)^{\prime}$ with respect to the topology of bi-bounded convergence on $E^{\prime} \otimes_{K} E$. In particular $F$ is a 
total set of continuous linear functionals on $E^{\prime} \otimes_{K} E$.

Proof. Let $\varphi \in D, a^{\prime} \in \check{E}$ be given, and let $f_{\varphi, a^{\prime}}$ be the function in $E_{K}^{\epsilon}(G, \check{E})$ corresponding to $F_{\varphi, a^{\prime}}$ under the isomorphism of Lemma 7.7. Then

$$
\begin{aligned}
\left\langle a, f_{\varphi, a^{\prime}}(x)\right\rangle & =F_{\varphi, a^{\prime}}\left(\epsilon_{x} \otimes a\right)=\left\langle m_{\epsilon_{x^{\prime}}}^{\#}(\varphi), a^{\prime}\right\rangle \\
& =\int_{K}\left\langle\delta_{K}(k) \epsilon_{x}(k \varphi) k^{-1} a, a^{\prime}\right\rangle d k \\
& =\left\langle a, \int_{K} \delta_{K}(k) \varphi(x k) a^{\prime} k^{-1} d k\right\rangle=\left\langle a,\left(\varphi \otimes a^{\prime}\right)^{\Gamma}(x)\right\rangle
\end{aligned}
$$

for all $a \in E$ and all $x \in G$. Hence

$$
f_{\varphi, a^{\prime}}=\left(\varphi \otimes a^{\prime}\right)^{\Upsilon} .
$$

Now $\check{E}$ is dense in $F$ so functions $\varphi \otimes a^{\prime}(\varphi \in D, a \in \check{E})$ span a dense linear sub. space of $\mathcal{D}(G, F)$ (see for instance the discussion in Grothendieck [7, Chapter II, p. 84]). Hence, by Lemma 7.9 the functions $f_{\varphi, a^{\prime}}=\left(\varphi \otimes a^{\prime}\right)^{\Pi}$ span a dense linear subspace of $D_{K}(G, F)$. Now the injection of $D_{K}(G, F)$ into $E_{K}(G, F)$ is continuous and onto a dense subspace. The denseness is most easily seen by invoking Lemma 7.2. It follows that the linear subspace $\left[f_{\varphi, a^{\prime}}: \varphi \in D, a^{\prime} \in \check{E}\right]$ (which clearly is contained in $D_{K}^{\epsilon}(G, \breve{E})$ ) is dense in $E_{K}(G, F)$ and then-a fortiori-in $E_{K}^{\epsilon}(G, \breve{E})$. The assertion now follows from Lemma 7.7. The proof is complete.

Corollary 7.12. The map $\Omega: E^{\prime} \otimes_{K} E \rightarrow E_{K}^{\prime}(G, E)$ is a continuous linear isomorphism onto a dense subspace.

Proof. Let $u=\Sigma S_{i} \otimes a_{i} \in E^{\prime} \otimes_{K} E$ and suppose $\Omega(u)=0$. Then for all $\varphi \in D$ and all $a^{\prime} \in \check{E}$ we have

$$
0=\left\langle\Omega(u) \varphi, a^{\prime}\right\rangle=\sum\left\langle m_{S_{i}, a_{i}}^{\#}(\varphi), a^{\prime}\right\rangle=\sum F_{\varphi, a^{\prime}}\left(S_{i} \otimes a_{i}\right)=F_{\varphi, a^{\prime}}(u) .
$$

Since $F=\left\{F_{\varphi, a^{\prime}}\right\}$ is total we must have $u=0$ and $\Omega$ is injective. The range of $\Omega$ is dense. In fact, by Lemma 7.1 it suffices to observe that $\left[m_{S, a}: S \in E^{\prime}\right.$, $a \in E]$ is dense in $E^{\prime}(G, E)$, which is well known. The proof is complete.

We now turn to the discussion of when $\Omega$ is bicontinuous, i.e. when the two spaces $E^{\prime} \bar{\otimes}_{K} E$ and $E_{K}^{\prime}(G, E)$ are isomorphic. As before let $E_{C}^{\prime}$ (resp. $\left.E_{C}^{\prime}(G, E)\right)$ denote the space of distributions (resp. $E$-valued destributions) with support in the compact set $C \subseteq G$. If $C_{1} \subseteq C_{2}$ then $E_{C_{1}}^{\prime} \hat{\otimes} E$ may be identified with a closed subspace of $E_{C_{2}}^{\prime} \hat{\otimes} E$. Let $H$ be the inductive limit of the spaces $E_{C}^{\prime} \hat{\otimes} E$ where $C$ runs through the family of compact subsets of $G$. $H$ is a strict inductive limit of complete spaces and therefore complete.

Lemma 7.13. The spaces $H$ and $E^{\prime}(G, E)$ are linearly and topologically isomorphic. 
Proof. Let $\left\{C_{n}\right\}$ be an ascending sequence of compact subsets of $G$ such that $U C_{n}=G$ and $C_{n}$ is contained in the interior of $C_{n+1}$. Since $D$ is a nuclear space we have a natural isomorphism $D^{\prime}(G, E) \cong D^{\prime}(G) \otimes E$ [28, Proposition 50.5]. For each compact $C \subseteq G$ the spaces $E_{C}^{\prime}(G, E)$ and $E_{C}^{\prime}(G) \hat{\otimes} E$ carry the relative topologies of $D^{\prime}(G, E)$ and $D^{\prime}(G) \hat{\otimes} E$ respectively. If we identify $E_{C}^{\prime}(G) \hat{\otimes} E$ with its image in $D^{\prime}(G, E)$ we clearly have

$$
E_{C_{i}}^{\prime}(G) \hat{\otimes} E \subseteq E_{C_{i}}^{\prime}(G, E) \subseteq E_{C_{i+1}^{\prime}}(G) \hat{\otimes} E
$$

since the topologies agree and $E^{\prime}(G, E)$ by definition is the inductive limit of $E_{C_{i}}^{\prime}(G, E), i=1,2, \ldots$, the lemma follows.

COROLLARY 7.14. If the inductive and the projective tensor product topologies agree on $E^{\prime} \otimes E$ then $E^{\prime} \bar{Q}_{K} E$ and $E_{K}^{\prime}(G, E)$ are topologically and linearly isomorphic.

Proof. $E^{\prime} \otimes_{\pi} E \cong E^{\prime} \otimes_{\epsilon} E$ since $E^{\prime}$ is a nuclear space. Hence, under the stated condition the identity map $E_{C}^{\prime} \otimes_{\epsilon} E \rightarrow E^{\prime} \otimes_{\beta} E$ is continuous for each compact $C \subseteq G$ : Hence we obtain a continuous linear map $\Phi$ of $H$ into $E^{\prime} \otimes E$ Now $\Phi$ restricted to $E^{\prime} \otimes E$ is injective and its inverse is continuous, so $\Phi$ is a topological isomorphism of $H$ onto $E^{\prime} \otimes E$. By Lemma 7.13 this implies that the natural injection $\Omega^{\prime}: E^{\prime} \otimes_{\beta} E \rightarrow E^{\prime}(G, E)$ is bicontinuous.

Let $N$ be the closed linear subspace of $E^{\prime} \otimes_{\beta} E$ generated by elements of the form $S k \otimes a-S \otimes k a\left(S \in E^{\prime}, a \in E, k \in K\right)$. By Proposition 2.3, we may identify $E^{\prime} \otimes_{K} E$ with $E^{\prime} \otimes_{\beta} E / N$, and we let $\gamma$ be the continuous open map of $E^{\prime} \otimes E$ onto $E^{\prime} \otimes_{K} E$ obtained this way, viz: $\gamma(S \otimes a)=S \otimes_{K} a$. The following diagram clearly commutes:

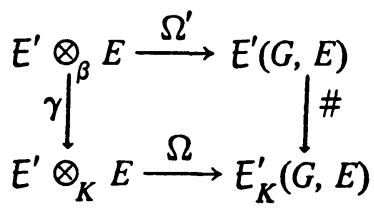

Since $\gamma$ is continuous, $\Omega^{\prime}$ is bicontinuous and \# is open, $\Omega$ is bicontinuous. The result follows.

LEMMA 7.15. If $E$ is either normable or the strong dual of a reflexive Fréchet space, then $E^{\prime} \otimes_{\beta} E \cong E^{\prime} \otimes_{\pi} E$.

Proof. $E^{\prime} \otimes_{\pi} E$ is in both cases a Mackey space [7, Chapter I, Corollary 1, p. 44], and the identity map $E^{\prime} \otimes_{\beta} E \rightarrow E^{\prime} \otimes_{\pi} E$ is continuous. Hence all we have to do is to show that if $\varphi$ is a continuous linear functional on $E^{\prime} \otimes_{\beta}$ $E$ then it is continuous on $E^{\prime} \otimes_{\pi} E$. If $E$ is a normed space or the dual of a 
reflexive Fréchet space this follows from [28, Theorem 48.1]. The proof is complete.

The proof of Proposition 6.2 is now clear. The isomorphism $\Omega$ intertwines the action of $G$ on ${ }^{G} E=E^{\prime} \bar{\otimes}_{K} E$ and $E_{K}^{\prime}(G, E)$. The equivalence of ${ }^{G} E$ and $E_{K}^{\prime}(G, E)$ as left differentiable $G$-modules follows directly from Corollary 7.14 and Lemma 7.15. This completes the proof of Proposition 6.2.

REMARK 7.16. It is clearly necessary to make some assumption on $E$ to have the topologies on $E \bar{\otimes}_{K} E$ and $E_{K}^{\prime}(G, E)$ identical. Indeed, by Lemma 7.13 and the diagram in Corollary 7.14 it is necessary that the identity map $E_{C}^{\prime} \otimes_{\epsilon} E$ $\rightarrow E^{\prime} \otimes_{\beta} E$ be continuous. Take $E=E$ and let $\omega: E^{\prime} \times E \rightarrow C$ be the canonical bilinear form, i.e. $\omega(S, \varphi)=S(\varphi)$. $\omega$ is hypocontinuous. Hence, if $i$ : $E_{C}^{\prime} \otimes_{\epsilon} E \rightarrow E^{\prime} \otimes_{\beta} E$ was continuous, the restriction of $\omega$ to $E_{C}^{\prime} \times E$ would be continuous. But this is impossible. Indeed, suppose there are neighbourhoods of $0, U$ in $E_{C}^{\prime}, V$ in $E$ such that $|\omega(U \times V)| \leqslant 1$. Then $U \subseteq V^{0}$, and $V^{0}$ is bounded in $E^{\prime}$. Hence $U$ is bounded in $E_{C}^{\prime}$ so $E_{C}^{\prime}$ is normable. But $E_{C}^{\prime}$ is also a nuclear space-hence finite dimensional, which is false.

8. The dual modules of ${ }^{G} E$ and $E^{G}$. Preserve the previous notation and conventions.

Proposition 8.1. Suppose that the following conditions are satisfied:

(a) Each bounded subset of $E^{\prime} \bar{\otimes}_{K} E$ is contained in the closed, balanced, convex hull of a set $A \otimes_{K} B$ where $A$ is bounded in $E^{\prime}$ and $B$ is bounded in $E$.

(b) Each compact subset of $E_{b}^{\prime}$ is equicontinuous.

(c) $E_{b}^{\prime}$ is complete.

Then

$$
\left({ }^{G} E\right)^{2} \cong(\check{E})^{G}
$$

as right differentiable G-modules. In terms of representations this means that

$$
\left({ }^{G} \pi\right)^{\llcorner} \cong(\check{\pi})^{G} \text {. }
$$

Proof. This is an immediate consequence of Lemma 7.7 since the map $F \rightarrow f$ intertwines the action of $G$.

Proposition 8.2. If $E$ is the strong dual of a reflexive Fréchet space then

(i) the conditions (a), (b) and (c) above are satisfied;

(ii) the inductive and the projective tensor product topologies on $E^{\prime} \otimes_{K} E$ coincide;

(iii) $E_{K}(G, \check{E})$ is a reflexive Frechet space with strong dual $E^{\prime} \bar{\otimes}_{K} E$;

(iv) if $E$ is also a nuclear space then $E^{\prime} \bar{\otimes}_{K} E$ and $E_{K}(G, \breve{E})$ are nuclear.

Proof. Let $Q$ be a bounded subset of $E_{K}^{\prime}(G, E)$. We claim that there is 
a bounded subset $Q^{\prime}$ of $E^{\prime}(G, E)$ such that $\left(Q^{\prime}\right)^{\#} \supseteq Q$. Indeed, $E_{K}^{\prime}(G, E)$ is a strict inductive limit so there is a compact set $C \subseteq G$ such that $Q$ is contained in $E_{C, K}^{\prime}(G, E)$. Let $\rho$ be the local inverse of \# defined in the proof of Lemma 7.2. $\rho$ is continuous so $Q^{\prime}=\rho(Q)$ is bounded in $E^{\prime}(G, E)$. The claim follows.

For the proof first observe that (ii) is an immediate consequence of Lemma 7.15 since $E^{\prime} \otimes_{K} E$ is the quotient of $E^{\prime} \otimes E$ modulo a closed subspace. We also know that if $E$ is the dual of a reflexive Fréchet space then (b) and (c) hold. Moreover, by the results of $\$ 7$ we may identify $E^{\prime} \bar{\otimes}_{K} E$ with $E_{K}^{\prime}(G, E)$, and obviously also $E^{\prime} \bar{\otimes} E$ with $E^{\prime}(G, E)$. Hence (i) follows from [7, Chapter I, Proposition 5, p. 43] and the observation above.

Next $E\left(G, E_{b}^{\prime}\right) \cong E(G) \hat{\otimes} E_{b}^{\prime}$ which is a reflexive Fréchet space [7, Chapter II, Corollary 1, p. 77]. Hence $E_{K}\left(G, E_{b}^{\prime}\right)$ is a reflexive Fréchet space. The dual of $E(G) \hat{\otimes} E_{b}^{\prime}$ is $E^{\prime} \hat{\otimes} E$ which therefore is reflexive. Hence the quotient $E^{\prime} \otimes_{K} E$ is evaluable. It is also semireflexive, for if $Q$ is a bounded, closed subset of $E^{\prime} \bar{\otimes}_{K} E$, let $Q^{\prime}$ be a closed bounded convex and circled subset of $E^{\prime} \bar{\otimes} E$ whose image under the quotient map contains $Q . Q^{\prime}$ is weakly compact, and the quotient map is weakly continuous, so $Q$ is relatively weakly compact. Hence $E^{\prime} \bar{\otimes}_{K} E$ is semireflexive, hence reflexive. Now, by (i) and Lemma 7.7 this implies (iii) since $E_{b}^{\prime}$ is complete.

Finally $E\left(G, E_{b}^{\prime}\right)$ and hence $E_{K}\left(G, E_{b}^{\prime}\right)$ is nuclear if $E$ (or $E_{b}^{\prime}$ ) is [7, Chapter II, Proposition 13, p. 76]). But then $E^{\prime} \bar{\otimes}_{K} E$, being the strong dual of a nuclear Fréchet space, is nuclear. This proves (iv) and completes the proof.

Proposition 8.3. Let $X$ be a right differentiable $K$-module, $X$ a reflexive Fréchet space. Then

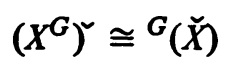

as left differentiable G-modules. Stated in terms of representations this means: If $\pi_{1}$ is a differentiable representation of $K$ on a reflexive Frechet space $X$, then

$$
\left(\pi_{1}^{G}\right)^{\sim} \cong{ }^{G}\left(\check{\pi}_{1}\right)
$$

Proof. Let $E=X_{b}^{\prime}$ and regard $E=X$ as a left differentiable $K$-module under the dual action. Then $X^{G}=(\check{E})^{G}=\left({ }^{G} E\right)^{-}$by (8.1). Now ${ }^{G} E$ is reflexive

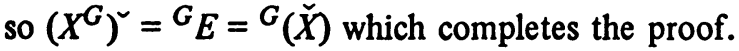

9. The Frobenius theorem of Bruhat. In [2] Bruhat gives a version of the Frobenius reciprocity theorem in terms of "formes d'entrelacement"-intertwining forms for two representations. His result is based on still another definition of induced representations. In this and the next section we discuss the relationship between his concept and ours, and show that his Frobenius result is 
a simple consequence of the tensor product machinery, modulo a realization which will be given towards the end of this section.

Let $\rho$ and $\lambda$ be continuous representations of a group $G$ in locally convex spaces $E$ and $F$ respectively. A hypocontinuous bilinear form $\omega$ on $E \times F$ is intertwining for $\rho$ and $\lambda$ if

$$
\omega(\rho(x) a, \lambda(x) b)=\omega(a, b)
$$

for all $x \in G, a \in E$ and $b \in F$. Clearly $\omega$ is intertwining for $\rho$ and $\lambda$ if and only if $\omega$ is $G$-balanced on $E \times F$ when we regard $E(F)$ as right (left) continuous $G$-modules. Indeed: $\omega(\rho(x) a, \lambda(x) b)=\omega\left(a x^{-1}, x b\right)$. We will therefore stick to our earlier practice, and formulate the result in terms of $G$ - (or $K \cdot$ ) balanced forms. Let $B_{G}(E, F)$ denote the space of hypocontinuous $G$-balanced bilinear forms on $E \times F$.

Let $K$ be a closed subgroup of the Lie group $G$, and let $\pi, \lambda$ be differentiable representations of $K, G$ on the complete locally convex spaces $E, F$ respectively. A functor $E \rightarrow E^{G}$ of $C_{l}^{\infty}(K)$ into $C_{l}^{\infty}(G)$ is called a quasi-adjoint for the restriction functor res: $F \rightarrow F_{K}$ of $C_{r}^{\infty}(G)$ into $C_{r}^{\infty}(K)$ if

$$
B_{G}\left(F, E^{G}\right) \cong B_{K}\left(F_{K}, E\right) \text {. }
$$

In this terminology Bruhat's Théorème 6.4 [2] shows the existence of a quasiadjoint in the categories of differentiable Fréchet modules.

We first show that if $E$ is the dual of a reflexive Fréchet space then our previous adjoint ${ }^{G} E=E_{K}^{\prime}(G, E)$ is in fact a quasi-adjoint.

Proposition 9.1. Suppose $E$ is the dual of a reflexive Fréchet space. Then $B_{G}\left(F,{ }^{G} E\right) \cong B_{K}\left(F_{K}, E\right)$.

Proof. $B_{G}\left(F,{ }^{G} E\right)=B_{G}\left(F, E^{\prime} \bar{\otimes}_{K} E\right)$ which by Proposition 8.2(i) and Lemma 2.8 is equal to

$$
\begin{aligned}
B_{G}\left(F, E^{\prime} \otimes_{K} E\right) & =\left(F \otimes_{G}\left(E^{\prime} \otimes_{K} E\right)\right)^{\prime} \\
& =\left(\left(F \otimes_{G} E^{\prime}\right) \otimes_{K} E\right)^{\prime} \\
& =\left(F \otimes_{K} E\right)^{\prime} \quad(\text { Lemma } 4.5 \text { and Proposition 2.7) } \\
& =B_{K}\left(F_{K}, E\right)
\end{aligned}
$$

which proves the assertion.

We now turn to the solution of the problem when $E$ and $F$ are Fréchet spaces. Let us regard $D$ as a subspace of $E^{\prime}$ under the identification $f \rightarrow S_{f}$, where $S_{f}(\varphi)=\int \varphi(x) f(x) d x \quad(\varphi \in E, f \in D)$. $D$ is a submodule of $E^{\prime}$ under the natural actions of $G$ and $K$ on $E^{\prime}$, viz.

$$
f k=f * \epsilon_{k} \quad(f \in D, k \in K),
$$




$$
x f=\epsilon_{x} * f \quad(f \in D, x \in G) .
$$

In its own topology $D$ is a complete $L F$-space and a differentiable $(G, K)$-bimodule with respect to these actions. This bimodule will be denoted by $D^{d}$. Let $D^{d} \otimes_{K} E$ denote the $K$-tensor product of $D^{d}$ and $E$ equipped with the inductive tensor product topology, and let $D^{d} \bar{\bigotimes}_{K} E$ be its completion. By similar arguments to those used in $\S 4$ we show that $D^{d} \bar{\otimes}_{K} E$ is a left differentiable $G$-module under the action $x(f \otimes a)=x f \otimes a$.

Proposition 9.2. Let $E(F)$ be a Fréchet space which is a left (right) differentiable $K(G)$-module. Then

$$
B_{G}\left(F, D^{d} \bar{\otimes}_{K} E\right) \cong B_{K}\left(F_{K}, E\right) .
$$

The proof of this result will be postponed till the end of this section. Let $D_{K}^{d}(G, E)$ denote the space of differentiable functions $f: G \rightarrow E$ satisfying the following two conditions:

$$
\text { supp } f \subseteq C K \text { for some compact subset } C \subseteq G \text {, }
$$

and

$$
f(x k)=\left(\delta_{K}(k) / \delta_{G}(k)\right) \pi\left(k^{-1}\right) f(x) \quad(x \in G, k \in K) .
$$

We equip $D_{K}^{d}(G, E)$ with the inductive limit topology as described in $\S 7$. $D_{K}^{d}(G, E)$ is made into a left differentiable $G$-module under the action $(x f)(y)=$ $f\left(x^{-1} y\right)(x, y \in G)$.

Proposition 9.3. If $E$ is a Fréchet space $D^{d} \bar{\otimes}_{K} E \cong D_{K}^{d}(G, E)$ as differentiable G-modules.

Proof. The proof of this result parallels the proof of Proposition 6.2 given in §7. We sketch the argument. First, if $f \in D(G, E)$ we define

$$
f^{\#}(x)=\int_{K} \frac{\delta_{K}(k)}{\delta_{G}(k)} k^{-1} f\left(x k^{-1}\right) d k
$$

and find that $f \rightarrow f^{\#}$ is a surjective continuous and open linear map of $D(G, E)$ onto $D_{K}^{d}(G, E)$. If $\varphi \in D, a \in E$ let $(\varphi \otimes a)(x)=\varphi(x) a(x \in G)$ and define the bilinear map $\omega_{1}(\varphi, a)=(\varphi \otimes a)^{\#}$. One shows that $\omega_{1}$ is a $K$-balanced hypocontinuous bilinear map of $D^{d} \times E$ into $D_{K}^{d}(G, E)$. Hence we obtain a continuous linear map $\Omega_{1}: D \otimes_{K} E \rightarrow D_{K}^{d}(G, E)$ with dense range. To show that $\Omega_{1}$ is injective we produce a separating family of continuous linear functionals $F_{S, a^{\prime}}\left(S \in E^{\prime}, a^{\prime} \in E^{\prime}\right)$ by computing the dual of $D^{d} \otimes_{K} E$.

By Proposition 2.6 we get $\left(D^{d} \otimes_{K} E\right)^{\prime} \cong D_{K}^{\prime}(G, E)$ since $E$ is a Fréchet space. It is now easy to show that if $F_{S, a^{\prime}}\left(\varphi \otimes_{K} a\right)=S\left(a^{\prime} \circ(\varphi \otimes a) \#\right)$ then the family $\left\{F_{S, a^{\prime}}\right\}$ will be separating, and we obtain that $\Omega_{1}$ is injective. To show 
that $\Omega_{1}$ is a homeomorphism first observe that $\mathcal{D}(G, E) \cong D(G) \bar{\otimes} E$ since $E$ is a Fréchet space [7, Chapter II, §3, p. 84]. The result now follows easily.

CoROLlaRY 9.4. If $E$ is a Fréchet space then $D^{d} \bar{\otimes}_{K} E$ is saturated, and the strong dual of $D^{d} \bar{\otimes}_{K} E$ is topologically and linearly isomorphic to $D_{K}^{\prime}\left(G, E_{b}^{\prime}\right)$.

Proof. We argue as in the proof of Proposition 8.2(i). If $Q \subseteq D_{K}^{d}(G, E)$ is bounded, then $Q$ is contained in $D_{K, C}^{d}(G, E)$ for some compact subset $C$. \# of the proof above has a continuous local inverse, so there is a bounded subset $Q_{1} \subseteq D_{C_{1}}^{d}(G, E)$ (for some compact $\left.C_{1} \subseteq G\right)$ such that $Q_{1}^{\#} \supseteq Q$. But $D_{C_{1}}^{d}(G, E)$ $\cong D_{C_{1}}^{d} \hat{\otimes} E$ which is saturated. The result now follows easily.

Observe that $D_{G}^{d}(G, E) \cong E$ as left differentiable $G$-modules. Indeed, the map $f \rightarrow f(e)$ is a topological isomorphism.

Proof of Proposition 9.2. We have

$$
B_{G}\left(F, D^{d} \bar{\otimes}_{K} E\right)=\left(F \bar{\otimes}_{G}\left(D^{d} \bar{\otimes}_{K} E\right)\right)^{\prime}
$$

which equals $\left(\left(F \bar{\otimes}_{G} D^{d}\right) \bar{\otimes}_{K} E\right)^{\prime}$ by Corollary 9.4 and Proposition 2.10. By the observation above (applied to $F$ ) and Proposition 9.3, $F \bar{\otimes}_{G} D^{d} \cong F$. Hence $B_{G}\left(F, D^{d} \bar{\otimes}_{K} E\right)=\left(F \bar{\otimes}_{K} E\right)^{\prime}=B_{K}\left(F_{K}, E\right)$. The proof is complete.

Bruhat's Frobenius result is now an immediate consequence. In fact, let $D_{K}^{B}(G, E)$ be the space of differentiable functions $f: G \rightarrow E$ with compact support modulo $K$ and satisfying

$$
f(x k)=\left[\delta_{K}(k) / \delta_{G}(k)\right]^{1 / 2} \pi\left(k^{-1}\right) f(x) \quad(x \in G, k \in K) .
$$

Then we get

Corollary 9.5 (BRUHAT). If $E$ and $F$ are Fréchet spaces, then the intertwining number of the representations of $G$ on $D_{G}^{B}(G, E)$ and $F$ is equal to the intertwining number of the representations $k \rightarrow\left[\delta_{K}(k) / \delta_{G}(k)\right]^{1 / 2} \pi(k)$ on $E$ and $\lambda_{K}$ on $F$.

Proof. Put $\pi_{1}(k)=\left[\delta_{K}(k) / \delta_{G}(k)\right]^{1 / 2} \pi(k)$ and apply Proposition 9.2 and 9.3 with respect to $\pi_{1}$.

10. Inducing in stages. Concluding remarks. Let $K \subseteq H \subseteq G$ where $K$ and $H$ are closed subgroups of $G$. Let $\pi$ be a differentiable representation of $K$ on a complete locally convex space $E$.

Proposition 10.1. We have $\left(\pi^{H}\right)^{G} \cong \pi^{G}$. If $E$ is the dual of a reflexive Fréchet space then ${ }^{G}\left({ }^{H} \pi\right) \cong{ }^{G} \pi$.

Proof. For the first equivalence we refer to the argument in [23, Theorem 4], which carries over without essential change. For the second equivalence 
we have ${ }^{G}\left({ }^{H} E\right)=E^{\prime}(G) \bar{\otimes}_{H}\left(E^{\prime}(H) \bar{\otimes}_{K} E\right)$. Now $E$ is the dual of a reflexive Fréchet space, which by Proposition 8.2(i) implies that $E^{\prime}(H) \bar{\otimes}_{K} E$ is saturated. $E^{\prime}(G) \bar{\otimes}_{H} E^{\prime}(H) \cong E^{\prime}(G)$ by Proposition $4.5(\mathrm{i})$, so by Proposition 2.10 we get ${ }^{G}\left({ }^{H} E\right) \cong E^{\prime}(G) \bar{\otimes}_{K} E={ }^{G} E$, which completes the proof.

REMARK 10.2. In [2, Proposition 4.2] Bruhat shows that inducing in stages goes through for his concept of induced representation, viz. the regular representation on $D_{K}^{B}(G, E)$.

We conclude with some remarks concerning the relationship between the various forms of induced representations discussed. In general they are all distict and have different universal properties. If $\delta_{K} / \delta_{G} \equiv 1$ and $G / K$ is compact we clearly have $D_{K}^{B}(G, E)=D_{K}^{d}(G, E)=E_{K}(G, E)$. Hence Bruhat's induced representation and our co-induced representation coincide. We have already seen that if $E$ is the dual of a reflexive Fréchet space, then the adjoint ${ }^{G} E$ is a quasiadjoint. It is interesting that in some cases the converse is true. Suppose $F=$ $X_{b}^{\prime}$ where $X$ is a barreled right differentiable $K_{G}$-module. Suppose $E$ is a quasiadjoint for the restriction functor. Then $L_{G}(G, F)=B_{G}(E, X)$ by Proposition 2.6, and this equals $B_{K}\left(E, X_{K}\right)=L_{K}\left(E, F_{K}\right)$. Hence in particular we get

Proposition 10.2. Let $\pi$ and $\rho$ be differentiable representations of $K$ and $G$ on Fréchet spaces $E$ and $X$ respectively. Let $\stackrel{G}{E}=D_{K}^{d}(G, E)$ and let $F=\check{X}$. Then

$$
L_{G}(\stackrel{E}{E}, F) \cong L_{K}\left(E, F_{K}\right)
$$

REMARK 10.3. As a curiosity one may observe that this result contains the fact that Haar measure on $G$ is unique. Indeed, let $K=\{e\}$ and $E=C=F$, with $\rho$ trivial. Then $L_{G}(D, \mathrm{C})=L(\mathrm{C}, \mathrm{C})=\mathrm{C}$ by Proposition 10.2. Similarly, Theorem 5.1 implies that there is essentially one $G$-invariant linear functional on $E^{\prime}(G)$, namely the function $\equiv 1$.

Let us finally disregard the action of $G$ and restate a few of the results concerning the duality between spaces of $E$-valued differentiable functions and $E$-valued distributions obtained on the foregoing pages. As before we assume that $E$ is a differentiable $K$-module.

Proposition 10.3. If $E$ is a reflexive Fréchet space then $E_{K}(G, E)$ and $D_{K}(G, E)$ are reflexive, and we have topological isomorphism

$$
\begin{aligned}
& E_{K}(G, E)^{\prime} \cong E_{K}^{\prime}\left(G, E_{b}^{\prime}\right), \\
& D_{K}(G, E)^{\prime} \cong D_{K}^{\prime}\left(G, E_{b}^{\prime}\right) .
\end{aligned}
$$

Proof. (10.2) follows from Propositions 6.2 and 8.2. (10.3) follows from Proposition 9.3 and Corollary 9.4. 


\section{REFERENCES}

1. J. F. Aarnes, A note on tensor products of vector spaces over Lie groups (to appear).

2. F. Bruhat, Sur les représentations induites des groupes de Lie, Bull. Soc. Math. France 84 (1956), 97-205. MR 18, 907.

3. - Distributions sur un groupe localement compact et applications à l'étude des représentations des groupes p-adiques, Bull. Soc. Math. France 89 (1961), 43-75. MR 25 \#4354.

4. G. Frobenius, Über Relationen zwischen den Characteren einer Gruppe und denen inrer Untergruppen, S.-B. Preuss. Akad. Wiss. 1898, 501-515.

5. I. M. Gel'fand, M. I. Graev and I. I. Pjateckii-Šapiro, Generalized functions. Vol. 6: Theory of representations and automorphic functions, "Nauka", Moscow, 1966; English transl., Saunders, Philadelphia, Pa., 1969. MR 36 \#3725; 38 \#2093.

6. R. Godement, $A$ theory of spherical functions. I, Trans. Amer. Math. Soc. 73 (1952), 496-556. MR 14, 620.

7. A. Grothendieck, Produits tensoriels topologiques et espaces nucléaires, Mem. Amer. Math. Soc. No. 16 (1955). MR 17, 763.

8. L. Gårding, Vecteurs analytiques dans les représentations des groupes de Lie, Bull. Soc. Math. France 88 (1960), 73-93. MR 22 \#9870.

9. Harish-Chandra, Representations of semisimple Lie groups. III, Trans. Amer. Math. Soc. 76 (1954), 234-253. MR 16, 11.

10. S. Helgason, Differential geometry and symmetric spaces, Pure and Appl. Math., vol. 12, Academic Press, New York and London, 1962. MR 26 \#2986.

11. E. Hewitt and K. A. Ross, Abstract harmonic analysis. Vol. I: Structure of topological groups. Integration theory, group representations, Die Grundlehren der math. Wissenschaften, Band 115, Academic Press, New York; Springer-Verlag, Berlin, 1963. MR 28 \#158.

12. J. L. Kelley, I. Namioka et al., Linear topological spaces, Univ. Ser. in Higher Math., Van Nostrand, Princeton, N. J., 1963. MR 29 \#3851.

13. A. A. Kirillov, Unitary representations of nilpotent Lie groups, Uspehi Mat. Nauk 17 (1962), no. 4 (106), 57-110 =Russian Math. Surveys 17 (1962), no 4, 53-104. MR 25 \#5396.

14. G. W. Mackey, On induced representations of groups, Amer. J. Math. 73 (1951), 576-592. MR 13, 106.

15. Induced representations of locally compact groups. I, Ann. of Math. (2)

55 (1952), 101-139. MR 13, 434.

16. - Induced representations of locally compact groups. II, Ann. of Math. (2) 56 (1953), 193-221. MK 15, 101.

17. S. Mac Lane, Categorical algebra, Bull. Amer. Math. Soc. 71 (1965), 40-106. MR 30 \#2053.

18. Categories for the working mathematician, Springer-Verlag, Berlin, 1971.

19. F. I. Mautner, Induced representations, Amer. J. Math. 74 (1952), 737-758.

MR 14, 134.

20. C. C. Moore, On the Frobenius reciprocity theorem for locally compact groups,

Pacific J. Math. 12 (1962), 359-365. MR 25 \#5134.

21. N. S. Poulsen, On $C^{\infty} \cdot$ vectors and intertwining bilinear forms for representations of Lie groups, J. Functional Analysis 9 (1972), 87-120. MR 46 \#9239.

22. M. A. Rieffel, Induced Banach representations of Banach algebras and locally compact groups, J. Functional Analysis 1 (1967), 443-491. MR 36 \#6544.

23. R. Rigelhof, Induced representations of locally compact groups, Acta. Math. 125 (1970), 155-187. MR 43 \#7550.

24. L. Schwartz, Théorie des distributions, Vol. I, Actualités Sci. Indust., no. $1091=$

Publ. Inst. Math. Univ. Strasbourg 9, Hermann, Paris, 1950. MR 12, 31.

25. - Théorie des distributions. Vol. II, Actualités Sci. Indust., no. $1122=$

Publ. Inst. Math. Univ. Strasbourg 10, Hermann, Paris, 1951. MR 12, 833.

26. - Théorie des distributions à valeurs vectorielles. I, Ann. Inst. Fourier

(Grenoble) 7 (1957), 1-141. MR 21 \#6534. 
27. Théorie des distributions à valeurs vectorielles. II, Ann. Inst. Fourier (Grenoble) 8 (1958), 1-209. MR 22 \#8322.

28. F. Trèves, Topological vector spaces, distributions and kernels, Academic Press, New York and London, 1967. MR 37 \#26.

29. G. Warner, Harmonic analysis on semi-simple Lie groups. I, Die Grundlehren der math. Wissenschaften, Band 188, Springer-Verlag, Berlin and New York, 1972.

30. D. P. Želobenko, Infinitely differentiable vectors in the theory of representations, Vestnik Moskov. Univ. Ser. I Math. Meh. 1965, no. 1, 3-10. (Russian) MR 32 \#5792.

DEPARTMENT OF MATHEMATICS, UNIVERSITY OF COLORADO, BOULDER, COLORADO 80302

DEPARTMENT OF MATHEMATICS, UNIVERSITY OF TRONDHEIM, TRONDHEIM, NORWAY (Current address) 